\title{
A Simplex Architecture for Intelligent and Safe Unmanned Aerial Vehicles
}

By

\author{
Prasanth Vivekanandan
}
Submitted to the Department of Electrical Engineering and Computer Science and the Graduate Faculty of the University of Kansas in partial fulfillment of the requirements for the degree of Master of Science

Prof.Yun,Heechul, Chairperson

Committee members

Prof.Kulkarni,Prasad

Prof.Luo,Bo

Date defended: 
The Thesis Committee for Prasanth Vivekanandan certifies that this is the approved version of the following thesis :

A Simplex Architecture for Intelligent and Safe Unmanned Aerial Vehicles

Prof.Yun,Heechul, Chairperson

Date approved: 


\begin{abstract}
Unmanned Aerial Vehicles (UAVs) are increasingly demanded in civil, military and research purposes. However, they also possess serious threats to the society because faults in UAVs can lead to physical damage or even loss of life. While increasing their intelligence, for example, adding vision-based sense-and-avoid capability, has a potential to reduce the safety threats, increased software complexity and the need for higher computing performance create additional challenges software bugs and transient hardware faults that must be addressed to realize intelligent and safe UAV systems.

In this thesis, we present a fault tolerant system design for UAVs. Our proposal is to use two heterogeneous hardware and software platforms with distinct reliability and performance characteristics: High-Assurance (HA) and High-Performance (HP) platforms. The HA platform focuses on simplicity and verifiability in software and uses a simple and transient fault tolerant processor, while the HP platform focuses on intelligence and functionality in software and uses a complex and high performance processor. During the normal operation, the HP platform is responsible for controlling the UAV. However, if it fails due to transient hardware faults or software bugs, the HA platform will take over until the HP platform recovers.

We have implemented the proposed design on an actual UAV using a low-cost Arduino and a high-performance Tegra TK1 multi core platform. Our case-studies show that our design can improve safety without compromising performance and intelligence of the UAV.
\end{abstract}




\section{Acknowledgements}

First and foremost I would like to thank Dr.Heechul Yun who is my advisor. This work was possible mainly because of his patience, motivation, enthusiasm, and immense knowledge. His guidance helped me in all the time of research and writing of this thesis.

Besides my advisor, I thank Dr.Shawn Keshmiri for his help and support. This is an inter disciplinary work involving Aerospace and Computer science and Dr.Keshmiri supported me by providing the required resource and advise for this work. I would like to thank Dr.Gonzalo Garcia for patiently answering all my questions on the autonomous controller design.

I would like to thank all the members of our research group Farzad Farshchi, Waqar Ali and Elise McEllhiney for being very friendly and helpful. A special thanks to Prathap Kumar Valsan for his support and valuable advice.

Most importantly, I would like to thank my family. I owe my parents a great debt of gratitude for teaching me valuable life lessons and always helped to keep my spirits up. 


\section{Publication}

This work has led to the following publication:

P. Vivekanandan, G. Garcia, H. Yun and S. Keshmiri, "A Simplex Architecture for Intelligent and Safe Unmanned Aerial Vehicles," 2016 IEEE 22nd International Conference on Embedded and Real-Time Computing Systems and Applications (RTCSA), Daegu, 2016, pp. 69-75. doi: 10.1109/RTCSA.2016.17 nominated for best student paper award 


\section{Contents}

1 Introduction 1

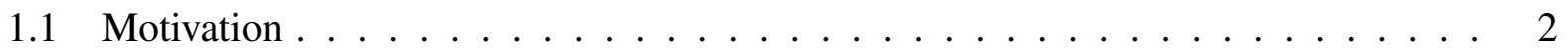

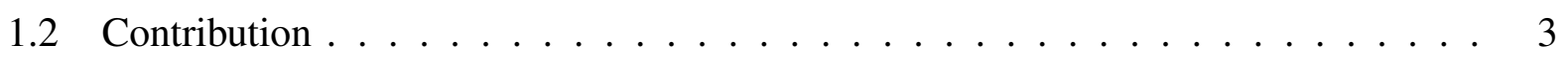

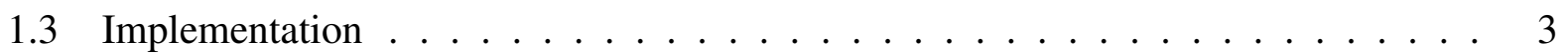

1.4 Thesis Overview $\ldots \ldots \ldots \ldots \ldots \ldots \ldots \ldots$

2 Background and Related Work 5

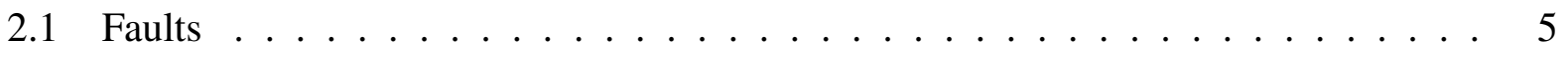

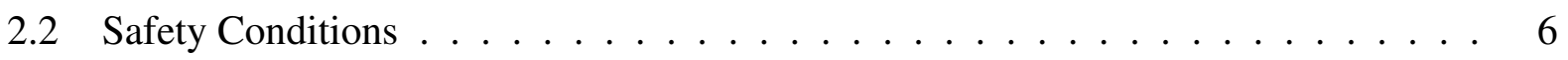

2.3 Related Work . . . . . . . . . . . . . . . . . . . . . . 7

3 UAV Simplex Architecture $\quad 10$

3.1 Fault Model . . . . . . . . . . . . . . . . . . . . . . . . . 12

3.2 Safety Region . . . . . . . . . . . . . . . . . . . . . 12

4 Prototype Avionics $\quad 14$

4.1 Hardware . . . . . . . . . . . . . . . . . . . . . . 14

$4.1 .1 \quad$ Sensors $\ldots \ldots \ldots \ldots \ldots \ldots \ldots \ldots \ldots$

4.1.2 High Performance Platform . . . . . . . . . . . . . . . 15

4.1.3 High Assurance Platform . . . . . . . . . . . . . . . . . . . 15

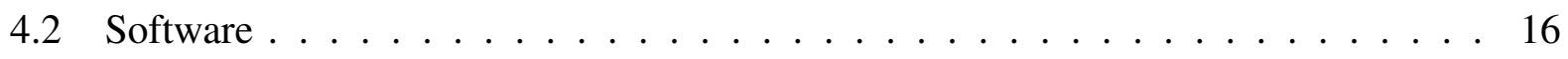


4.2.1 High Performance Controller . . . . . . . . . . . . . . . . . . . . 16

4.2 .2 High Assurance Controller . . . . . . . . . . . . . . . . . . . . 16

4.2 .3 Decision Logic . . . . . . . . . . . . . . . . . . . . . 17

4.3 Fault Detection and Recovery . . . . . . . . . . . . . . . . . . 17

$4.3 .1 \quad$ Safety Region . . . . . . . . . . . . . . . . . . . . 20

4.3.2 Decision Logic Conditions . . . . . . . . . . . . . . . . . . . 21

4.3.2.1 Condition 1: Valid Controller Output . . . . . . . . . . . 21

4.3.2.2 Condition 2: Kalman Filter Divergence . . . . . . . . . . . 22

4.3.2.3 Condition 3: Sensor Communication with the Performance Con-

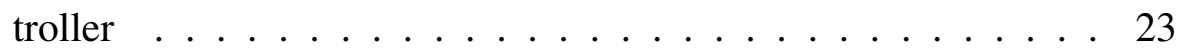

4.3.2.4 Condition 4: Stall Speed Condition . . . . . . . . . . . 23

4.3.2.5 Condition 5: FAA Regulation on Altitude . . . . . . . . . 24

5 Evaluation $\quad 25$

$5.1 \quad$ HiL Test . . . . . . . . . . . . . . . . . . . 25

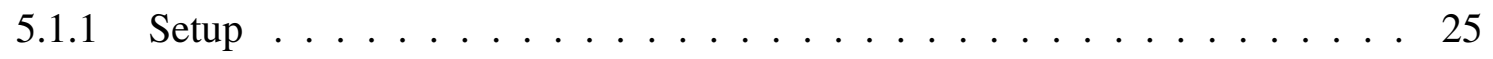

5.1.2 Case Study: Performance Controller Crash _ . . . . . . . . . . . . 26

5.2 Flight Test . . . . . . . . . . . . . . . . . . . . . . . . . . . 29

6 COTS Autopilot with UAV Simplex Architecture 34

6.1 Implementation . . . . . . . . . . . . . . . . 35

6.2 HiL Test . . . . . . . . . . . . . . . . . . 36

6.3 Aircraft Setup using Pixhawk and On Board Computer . . . . . . . . . . . . 37

$\begin{array}{lll}7 & \text { Conclusion } & 39\end{array}$

$\begin{array}{ll}\text { A Videos } & \mathbf{4 4}\end{array}$

B Issues Faced and Solved $\quad 45$

B.1 Divide by Zero $\ldots \ldots \ldots \ldots \ldots \ldots \ldots \ldots \ldots \ldots \ldots \ldots \ldots$ 


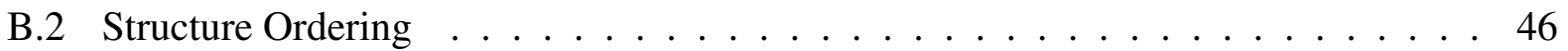

B.3 USB Hub Power Issue $\ldots \ldots \ldots$

$\begin{array}{ll}\text { C Avionics Installation } & 49\end{array}$

C.1 Software Dependencies . . . . . . . . . . . . . . . . . . . . . . . . . 49

C.2 Installing our Avionics Code $\ldots \ldots \ldots$. . . . . . . . . . . . . 49

D Source Code Listing

D.1 Controller Function Code . . . . . . . . . . . . . . . . . . . . . 51

D.2 Sensor Data Acquisition Code $\ldots \ldots \ldots \ldots$

D.2.1 RC, Air Velocity and Battery Voltage . . . . . . . . . . . . . 64

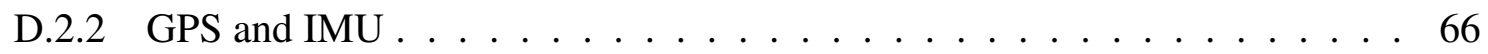




\section{List of Figures}

1.1 DG 808S with our custom built avionics . . . . . . . . . . . . . . . . 4

4.1 UAV simplex architecture . . . . . . . . . . . . . . . 15

4.2 ROS based software architecture on the highperformance platform (Tegra K1) . . . 17

4.3 Autonomous controller block diagram . . . . . . . . . . . . . . . . . . 18

4.4 Decision logic on Arduino. . . . . . . . . . . . . . . . . . . . . . . 19

5.1 Experiment setup for UAV simplex . . . . . . . . . . . . . . 26

5.2 The flight path . . . . . . . . . . . . . . . . . 27

5.3 Outputs of safety controller, performance controller, and decision logic. A fault is injected at time 100 second. . . . . . . . . . . . . . . . 28

5.4 Accelerator values measured from flight test, X-axis: Time in seconds, Y-axis: Acceleration in $\mathrm{G} \ldots \ldots \ldots \ldots$

5.5 Aircraft orientation in Euler angles measured from flight test, X-axis: Time in seconds, Y-axis: Euler angles in degrees . . . . . . . . . . . . . . . 30

5.6 Body rates measured from flight test, $\mathrm{X}$-axis: Time in seconds, Y-axis: Body rates

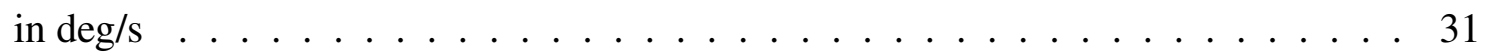

5.7 Aircraft velocity measured from flight test, $\mathrm{X}$-axis: Time in seconds, Y-axis: ve-

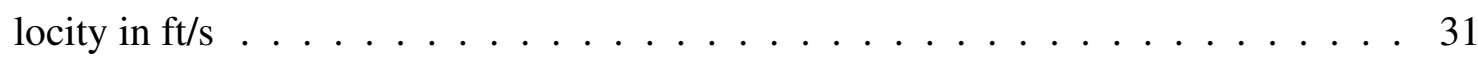

5.8 Rosgraph plot showing the nodes and topics and for the switch between controllers

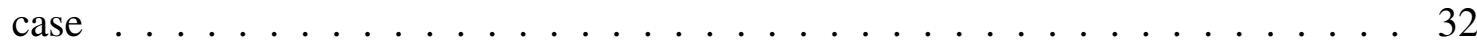


6.1 Pixhawk outer view . . . . . . . . . . . . . . . . . . . . 35

6.2 ROS nodes outline for PX4 and the controller program $\ldots \ldots \ldots 36$

6.3 Hardware in the Loop for PX4 as the safety platform $\ldots \ldots$. . . . . . . . 37

6.4 Skyhunter using Pixhawk and odroid avionics $\ldots \ldots \ldots$

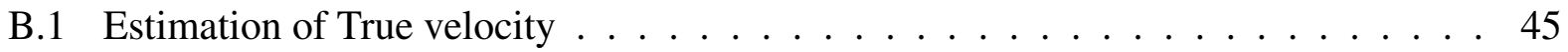

B.2 Matlab code to convert GPS position from global to local . . . . . . . . . . 46

B.3 Matlab generated $\mathrm{C}$ code to convert GPS position from global to local . . . . . 47

B.4 Picture of our avionics containing a usb hub $\ldots \ldots \ldots \ldots$ 


\section{List of Tables}

3.1 Platform characteristics $\ldots \ldots \ldots \ldots \ldots$

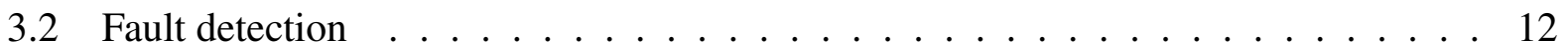




\section{Chapter 1}

\section{Introduction}

The use of Unmanned Aerial Vehicles (UAVs) is rapidly increasing in recent years due to diverse recreational, commercial, and military applications. Modern UAVs have begun incorporating a range of new technology, including electronic sensors that stabilise them. Some models can even be programmed to fly set paths or patterns. Another good example is a UAV with a small camera attached to it that gives the birds-eye view of the surroundings.

These advancements have not only seen UAVs becoming popular amongst even the most casual of hobbyists, but also in commercial endeavours. Large companies such as Facebook, Google and Amazon have already invested heavily in research related to drones, and even smaller companies such as florists, pizza restaurants (and even real-estate agencies) have started to investigate how drones can be used in their industry. More recently news agencies have begun to look at using drones when covering certain news stories, and Hollywood has already started using drones during the production of movies and TV shows.

Most of the UAVs work in close proximity with the humans and the physical environment. Hence, there is an increasing demand for intelligent UAV systems that are cognizant of the surrounding environment and perform sophisticated tasks including collision avoidance.

An intelligent UAV system requires integration of advanced sensor packages (e.g. vision) and high computational performance to process the enormous amount of real-time sensor data and to 
execute complex algorithms in a timely manner. The rapidly increasing computing capacity of modern embedded computing platforms multiple CPU cores, GPU, and other accelerators makes it feasible to develop such a UAV system while satisfying size, weight, and power (SWaP) requirements of UAVs. In order to get the full benefits of this powerful hardware, a powerful software frame work like CUDA for GPU applications, opencv for vision based application and so on is required. The combination of powerful hardware together with the powerful software makes the computing platform in the UAV to look almost like a desktop computer.

\subsection{Motivation}

The push for higher intelligence in UAV systems also creates serious side effects in terms of safety and reliability. First, the complexity of software systems is rapidly increasing, which makes it difficult to weed out software bugs. For example, an intelligent flight control system with vision based collision avoidance capability, which in itself can be complex and difficult to verify, may also depend on complex middleware packages (e.g., Robot Operating System, ROS [32]) and the OS (e.g., Linux), each of which may be comprised of multi-million lines of code.

Second, to achieve high intelligence, the use of high performance computing platforms is necessary. However, high performance computing platforms are increasingly prone to transient hardware faults (soft errors) due to environmental effects such as single-event upsets (SEUs) [15]. SEUs are caused by high energy particle strikes from cosmic rays [39] which result in bit flips. The technology treads to develop efficient and high-performance processors shrinking dimensions and operating voltage, and increased frequency and density have dramatically increased the possibilities of SEUs [15], which could result in unexpected failures in the system [23, 36]

There has been a large body of research in the control systems community regarding the design of fault tolerant control of UAV systems [34]. While these fault tolerant controllers are designed to handle structural damage, actuator and sensor failures, they typically do not handle system-level failure such as on-board computing platform malfunction, which prevents execution of the control algorithms in the first place. System-level reliability can be generally improved by redundancy. A 
well-known technique is triple modular redundancy (TMR) [26] in which three identical systems produce control outputs in order to survive from failures of any one of the system. However, the size, weight, and power considerations as well as the cost make the TMR solution undesirable, especially in small UAVs.

\subsection{Contribution}

To address the safety and reliability challenges of UAV systems, we present a UAV system design and implementation based on Simplex architecture [35]. The main idea of the Simplex architecture is that a simple verifiable controller provides safety of the system, while a complex, highperformance controller strives to achieve high system performance. The choice between the two controllers is determined by a decision module, which constantly assesses the safety status of the system and makes the decision.

Our contribution is a novel application of the Simplex architecture to develop both intelligent and reliable UAV systems in a cost effective manner. Specifically, we realize the Simplex architecture using two heterogeneous hardware platforms with distinct reliability and performance characteristics. The idea is that we use a reliable but less performance hardware platform, which we call a High-Assurance (HA) platform, to be responsible for safety while we use a more performance, but potentially less reliable platform, which we call a High-Performance (HP) platform, for performance and intelligence of the UAV. Only the HA platform both software and hardware forms the trusted computing base (TCB) of the UAV. The HP platform, on the other hand, can be affected by software bugs and SEUs, which could result in failures (e.g., crashes), but the whole system safety will still be insured by the safety platform.

\subsection{Implementation}

We applied the proposed design in implementing a custom fixed-wing UAV system, shown in Figure 1.1, using a low-cost Arduino as our HA platform and a Tegra TK1 as HP platform. We 


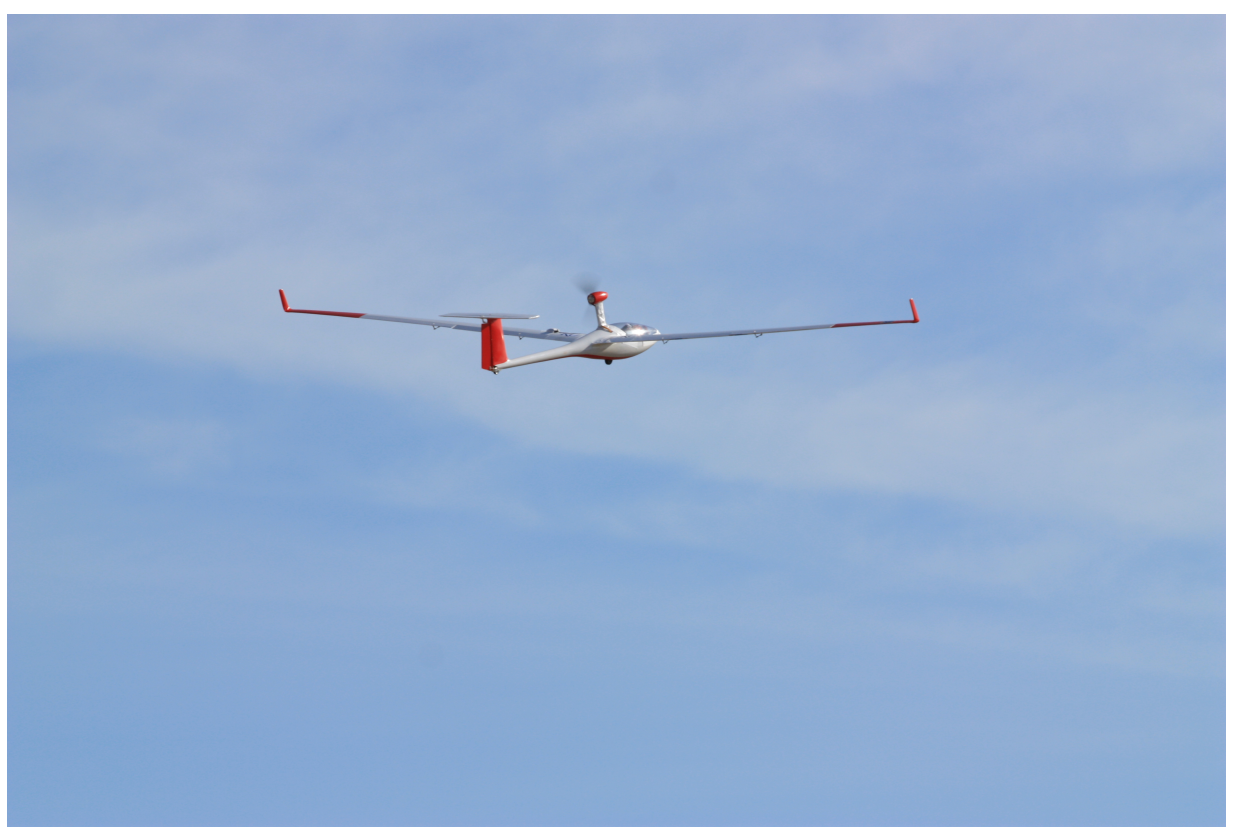

Figure 1.1: DG 808S with our custom built avionics

demonstrate the ability to recover from crashes through a set of fault-injection experiments in a hardware-in-the-loop simulation setup. The results suggest that the proposed design can substantially increase safety of intelligent UAVs.

\subsection{Thesis Overview}

The rest of the thesis is organized as follows. Chapter 2 discusses various fault types in a UAV and related background. Chapter 3 presents the proposed UAV simplex architecture. Chapter 4 describes our implementation of a fixed-wing UAV based on the proposed architecture. Chapter 5 presents our evaluation results and we conclude in Chapter 6. 


\section{Chapter 2}

\section{Background and Related Work}

In this section, we discuss various types of faults that can occur in a UAV and safety conditions that should be followed by the aircraft in order to be safe and reliable. We then discuss related work.

\subsection{Faults}

An UAV is a cyber-physical system, which includes cyber part (computing hardware and software) and physical part (sensors, actuators, UAV frame, and etc).

Faults in physical components of an UAV and their handling has been well studied in the control and aerospace communities. For example, structural damage or bias in sensor readings can be tolerated by advanced adaptive control algorithms that take such effects into account. A comprehensive review on the topic can be found in [34]. However, advanced control algorithms must be realized in software binaries running on computing platforms i.e., the cyber system.

Faults in the cyber system can arise from a number of different reasons. The most common type of faults are logical faults in software i.e., software bugs. As the demand for higher intelligence and functionality increase, the size and complexity of flight control software keeps increasing [20]. While model-based design and verification methodologies have made great progress $[21,20,16]$, it is still difficult to completely weed out all bugs. 
Another type of faults are temporal faults, which occur when the real-time requirements (i.e., deadlines) of various tasks in the system are not met. Temporal correctness is difficult to guarantee especially in multi core architecture because of uncontrolled sharing of many performance critical hardware resources such as cache and DRAM among concurrently executing tasks can cause highly variable timing $[25,30]$.

Third, often overlooked but increasingly important type of faults are transient hardware faults due to single event upsets (SEUs). SEUs are caused by cosmic radiation and alpha particles [39, 27] resulting bit flips. Because SEUs can occur anywhere in SRAM, registers, and combinational logic, it can cause, for example, an invalid instruction exception, a parity error, a memory access violation, a wrong conditional branch, and ultimately a system crash [36]. As technology scaling continues (i.e., more transistors in a chip), sensitivity to radiation has dramatically increased [15]. Traditional circuit-level solutions_-special circuit design and manufacturing process_-are not only expensive but also often lag several generations behind the state-of-the-art processors [18]. This is a serious impediment to develop intelligent UAVs that require cutting edge high performance computing capabilities.

\subsection{Safety Conditions}

In a UAV, the controller program acts in conjunction with the physical dynamics of the system. Based on the dynamics, the system has safety conditions that needs to be respected to maintain the safety of the UAV. Aircraft stall is one example for the safety condition [2]. When an aircraft flies at a certain altitude, it makes an angle called angle of attack with the wind. If the autonomous controller gives output such that the aircraft starts to climb then this angle of attack increases. Once the angle of attack crosses the threshold value (varies from one aircraft to another and it is determined using the aircraft's dynamics), the wings of the aircraft will not be able to lift the aircraft.

Another safety condition that needs to be respected by the UAV system is the regulations. The UAVs operate at close proximity with humans and physical environment. The government makes 
the regulations that must be followed by commercial UAVs. One such regulation is the limit on the altitude that commercial UAVs should fly. In United States the commercial UAVs are allowed to fly less than 400 feet from ground level [3].

In summary, as the demand for higher intelligence in UAVs increases, the importance of reliable computing software and hardware platform the cyber system increases. However, the increased software complexity and the use of high performance multi core processors, while necessary, would increase all three types of faults logical, temporal, and transient hardware faults of the cyber system of an UAV. The UAV systems face safety threats not only from these faults but also from the autonomous controller that is not obeying the physical constraints.

\subsection{Related Work}

Software reliability have long been a major concern in safety-critical cyber-physical systems. For example, software in commercial airplanes must follow certain standards (e.g., DO-178C [33] and ARINC-653 [13]) and be certified by certification authorities. At the application-level, modelbased designs and formal-methods [20, 16, 21] have made significant advance, it is still difficult to verify the correctness of the software at the code-level [28]. Furthermore, the applications also rely on many other system software components, including the OS and middleware, each of which may contain bugs.

System-level reliability and fault-tolerance techniques, especially in the context of intelligent cyber-physical systems, have been studied to mitigate possible software bugs. SAFER is a middleware framework that uses software-level redundancy to enhance system-level reliability. However, such a middleware solution may not survive from an OS failure (e.g., BSOD). The seL4 is a microkernel where its functional correctness was formally verified [24]. However, functional correctness does not means its temporal correctness is guaranteed. Nor it guarantees correct operations in the presence of transient hardware faults (SEUs), which are becoming more prevalent as technology scaling continues [18]. C'Mon focuses on detecting OS-level timing faults (e.g., deadline misses due to scheduling) that are caused by SEUs [36]. To guard the OS code and data against SEUs, 
dOSEK proposed to use a special encoding in storing OS code and data, which enables detection and recovery from the SEUs [23]. However, its software-based approach comes at a significant performance penalty.

SEUs can be reduced at each hardware component-level by, for example, adopting ECC and other so called "hardening" techniques [15]. Also, hardware redundancy solutions, such as Triplemodular redundancy (TMR) [26] or dual-redundancy, can protect the system from transient hardware faults [15]. In today's commercial airplanes and satellites, the physical redundancy based techniques are used due to their high safety-critical requirements [19]. However, these hardware techniques comes at substantial space and performance penalties and high cost and development time- unsuitable for UAVs. Also, the processors used in these applications often several generations behind the stat-of-the-art COTS processors $[25,17]$. This is a serious problem for intelligent UAVs where high computing performance is a key to achieve high intelligence.

The simplex architecture [35] is a special form of redundancy in which safety and performance are decoupled to safety and performance controllers, respectively. It was originally implemented at the application software-level in a single computing platform and used to upgrade/verify controllers without stopping the system [35]. More recently, Bak et al. implemented the safety-critical parts of the system - safety controller and decision logic-in FPGA so that it can tolerate systemlevel faults in the computer system running the performance controller for an inverted pendulum and a cardiac pacemaker [14]. [10] is an extension of simplex architecture and Abdi et al showed that a software fault in cyber-physical system can be recovered by restarting the system at runtime. In [12], Abdi et al proposed a novel approach to design a controller that enables the system to restart and remain safe during and after the restart. Their approach is also based on simplex architecture and it requires only one processing unit. Hence, [12] is almost similar to the original simplex architecture, but it also provides recovery from system level faults. Another version of simplex is shown in [38] and it is used to detect intrusion in cyber-physical systems. Abdi et al in [11] proposed a version of simplex architecture that adds security to the cyber-pyhsical systems by adding a monitoring module(checks for any intrusion) inside complex system. If monitoring 
module fails to detect any security failures, the periodic restart protects the system. 


\section{Chapter 3}

\section{UAV Simplex Architecture}

In this chapter, we review the Simplex architecture [35] and describe our two heterogeneous platforms based approach.

The Simplex architecture is composed of three components: a safety controller, a performance controller, and a decision logic [35]. Normally, the performance controller drives the plant (in our case, the UAV) as it offers higher control performance. However, if the safety conditions of the plant are to be violated, as determined by the decision logic, the safety controller will assume the control of the plant until the performance controller is recovered (for example, by restarting it). In this way, faults in the performance controller does not cause safety failure of the system.

In the original Simplex applications, however, all three components share the same computing hardware (processor) and software platform (OS, middleware) [35]. This means that system-level faults in the shared hardware and software platform-SEUs in the processor, bugs in the OS and middleware-could still compromise the safety of the system. as shown in Table 3.1. To overcome the limitations, we propose to realize the Simplex architecture by using two platforms with distinct reliability and performance characteristics, as shown in Table 3.1. The High-Assurance (HA) platform focuses on safety and verifiability over performance and functionality. For hardware, we assume that it uses chips that are more tolerant to SEUs. While a number of techniques can be used to reduce the SEU rate of a chip—ECC, manufacturing process, and etc.-using simple, low- 
Table 3.1: Platform characteristics

\begin{tabular}{c|c|c}
\hline Platform & High-Assurance (HA) & High-Performance (HP) \\
\hline $\begin{array}{c}\text { Hardware } \\
\text { Software }\end{array}$ & SEU resistant & SEU susceptible \\
Verifiable & Unverifiable \\
\hline
\end{tabular}

density chips running at low operating frequency could also help reduce the overall SEU rate [15]. For software, we assume that the platform uses a small RTOS with proven (verified) reliability. For example, the seL4 micro-kernel's functional correctness was formally verified [24]. Also, there are many other commercial/open-source RTOSs that have been used in critical applications.

On the other hand, the High-Performance (HP) platform focuses on performance and functionality over safety. For hardware, it may use a complex, high-performance (multicore) processor, which runs at multi-gigahertz frequencies and is composed of multi-billion transistors. Generally, such a processor suffers more SEUs than a simpler, low-performance one because higher density and operating frequency negatively affect SEUs [15]. For software, it may use a rich OS (e.g., Linux) and middleware solutions (e.g., Robot Operating System: ROS) to offer sophisticated capabilities needed to implement high intelligence. However, each of the software package may be comprised of many million lines of code, which makes it very difficult, if not impossible, to verify its correctness.

Among the three components in the Simplex architecture, we assign the safety controller and the decision logic on the HA platform, while assign the performance controller on the HP platform.

The performance controller may implement an advanced control algorithm and intelligent sensing capabilities, such as vision-based collision avoidance, that require high computing performance and rich OS and middleware support. The HP platform offers such computing power and functionality but it does not guarantee safety and reliability. On the other hand, the safety controller implements a simple and proven control algorithm that is always ready to take over the performance controller, if necessary, as determined by the decision logic. In our design, as in the original Simplex architecture, correct functioning of the safety controller and the decision logic is required. In addition, the HA platform provid es a safe and reliable execution environment although it may 
Table 3.2: Fault detection

\begin{tabular}{r|l}
\hline Observed behaviors & Faults in the HP platform \\
\hline No output & OS/controller crash \\
Delayed output & Deadline miss \\
Unsafe output & Bugs, SEUs, bad controller design, etc. \\
\hline
\end{tabular}

not provide high computing performance or rich middleware support.

\subsection{Fault Model}

We assume that faults can occur only in the HP platform. In other words, we trust the correct functioning of the HA platform the safety controller and decision logic. To ensure this, hardware of the HA platform must be resistant to transient hardware faults, and the complexity of its software must be limited to a level that can be rigorously tested and possibly verified. On the other hand, we do not trust both hardware and software of the HP platform-i.e., the OS can crash, the performance controller may crash or produce invalid outputs (e.g., NaN output) or simply miss the deadline due to resource contention. Also, the HP platform can suffer transient hardware faults (SEUs) which lead to system crash or application failure.

Lastly, in this work, we do not consider physical faults, such as structural damage, bias in sensor readings, sensor and actuator malfunction, and so on. We assume that these physical issues are tolerated by adaptive control algorithms, which have been extensively studied in the aerospace engineering community [34].

\subsection{Safety Region}

Physical systems, especially UAVs, have limits and constraints that need to be respected. For example, a UAV should have angle of attack less than critical angle of attack [2] to avoid stall condition. Safety region $S$ is a subset of State Space Model SSM where all this constraints are respected. 


$$
S S M=A x+B u
$$

$$
S \subseteq S S M
$$

Where $x$ denotes the states of the system and $u$ represents the inputs to the system. $A$ and $B$ matrices are the dynamics of the system. The safety region can be defined by the following equation.

$$
S=\left\{x \mid S_{x} \cdot x \leq 1\right\}
$$

Equation 3.3 conveys that for every state $x$ in the system state space model SSM there exists a safety matrix $S_{\mathrm{x}}$ such that dot product of safety matrix and their corresponding state should be less than or equal to one. If the system state is outside this safety region then the system is said to be unsafe.

Safety region also considers the limits on the actuators. The actuators have their range for operation. Hence this range should also be considered while defining the safety region. The safety region that defines this operating range for the actuators are formulated as follows.

$$
S_{u}=\left\{u \mid S_{u} \cdot u \leq 1\right\}
$$

Equation 3.4 conveys that, for every actuator value generated by the controller algorithm or the input to the system $u$ in the system state space model, there exists a safety matrix $S_{\mathrm{u}}$ such that dot product of safety matrix and their corresponding system input should be less than or equal to one. If the controller generates the value to the actuators outside this region, then actuators will not be able to handle them. 


\section{Chapter 4}

\section{Prototype Avionics}

We have designed an avionics based on the UAV simplex architecture. In this chapter, we discuss about the hardware and software that are used in our custom built avionics.

\subsection{Hardware}

\subsubsection{Sensors}

We categorize sensors into two groups: basic and advanced sensors. Basic sensors include GPS/IMU, airspeed, and pressure sensors and they are essential to flight. For GPS/IMU, we use a VectorNav VN-200 [8] module. For airspeed and pressure, we use an AMS 5812 pressure sensor and a pitot tube. The basic sensors are shared by both safety and performance controllers. On the other hand, advanced sensors are only used by the performance controller and considered not essential to flight although they may increase performance of the system. The advanced sensors include, for example, radars and cameras, which can be used to implement advanced sense-and-avoid capabilities. We are currently implementing a vision and radar based sense and avoid system. 


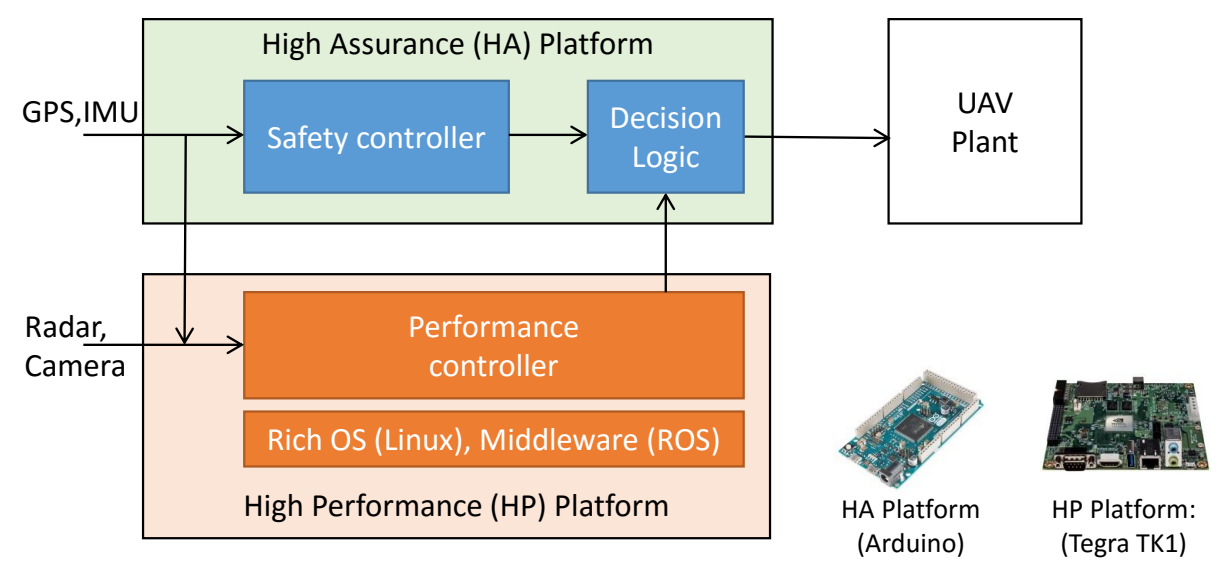

Figure 4.1: UAV simplex architecture

\subsubsection{High Performance Platform}

The HP platform is responsible for real-time processing of advanced sensors (e.g., vision and radar) and sophisticated control algorithms to achieve high control performance and sense-andavoid capabilities. To satisfy the performance demand, we use Nvidia's Tegra K1 processor, which equips four ARM Cortex-A15 cores, running at 2.3 GHz, and 192 Kepler based GPU cores [31].

\subsubsection{High Assurance Platform}

The HA platform is ultimately responsible for safety of the system and therefore must be simple and highly resistant to transient hardware faults. For this, we use an Arduino Due platform. The platform equips a single-core Cortex-M3 processor with $80 \mathrm{MHz}$ maximum operating frequency. It is based on simple in-order architecture and the number of transistors of the chip is much smaller than that of the Tegra K1. These characteristics make the HA platform less susceptible to SEUs. Also, the platform supports numerous I/O options GPIO, PWM, I2C, and etc that are needed to connect various basic sensors. 


\subsection{Software}

\subsubsection{High Performance Controller}

The performance controller is implemented on the Tegra K1. We use Ubuntu 12.04 Linux as the OS and the Robot Operating System (ROS) as the middle ware framework. In ROS, the system, a robot, is composed of a set of nodes, each of which is a separate Linux process participating in the communication network for the robot. A node can publish messages to a communication channel, called a topic, which can be subscribed by other nodes to receive the published messages. Figure 4.2 shows the nodes and topics of the performance controller. In the figure, the controller DG808 node is the main control node. The node subscribes the /vectornav/ins topic, to receive GPS and IMU sensor values, and the /arduino/pwm, to receive commands from the remote controller (RC) and pressure sensor values. (The RC is used for manual control of the UAV). The controller node publishes control outputs to the servo_op topic, which is then subscribed by the arduino node and is forwarded to the HA platform via serial. The MICORHARD ground station node is responsible for communicating with the remote ground station on the laptop PC. We use the open-source Qgroundcontrol [6] as the ground station software. We use a microhard modem for long range communication [4] between the UAV and the ground station to send/receive statistics (UAV to groundstation) and waypoints (groundstation to UAV) Currently, the performance controller only uses the basic sensors, and it does not utilize radar and vision sensors; we are actively developing radar and vision-based sense-and-avoid capability in the performance controller

\subsubsection{High Assurance Controller}

The safety controller runs on the Arduino Due platform. The control algorithm is a simple PID based one [22] and it is modeled and validated using Matlab Simulink [7]. Figure 4.3 shows the simulink model. We then generate $\mathrm{C}$ code of the controller using Matlab Simulink Coder. In this way, we minimize the possibility of bugs in the safety controller, although it does not guarantee the absence of bugs in the model. In fact, our initial model contained a divide zero bug, which was 


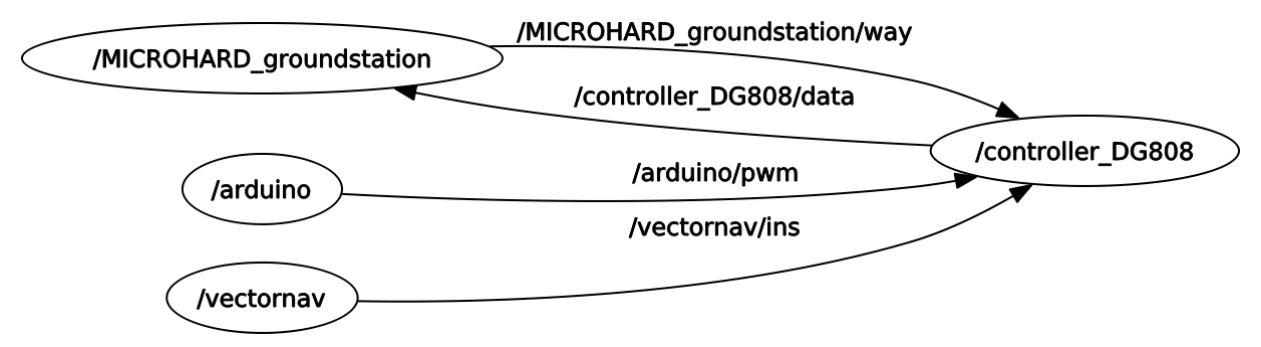

Figure 4.2: ROS based software architecture on the highperformance platform (Tegra K1)

manifested only in a certain sensor input value ranges. Nevertheless, model-based design is highly desired for a safety controller as it can significantly reduce the possibility of coding mistakes and other common software bugs $[20,16]$.

\subsubsection{Decision Logic}

The decision logic also runs on the Arduino Due, along with the safety controller. In fact, the generated $\mathrm{C}$ code of the safety controller is merged with the decision logic. The safety controller is directly called by the decision logic while the output of the performance controller is received asynchronously from the Tegra K1 via serial. Which output is used to actuate the UAV is then determined by the decision logic. Figure 4.4 shows the overall process of the decision logic. Note that each loop is executed periodically at a regular interval $(20 \mathrm{~Hz}$ in our current implementation). The implementation assumes that the safety controller always completes within the interval without errors-i.e., out_hap is always valid and computed within the interval (deadline). On the other hand, the output of the performance controller can be deemed unsafe or invalid due to a number of reasons.

\subsection{Fault Detection and Recovery}

The decision logic detects faults in the HP platform by observing its outputs (See Line 14 in Figure 4.4). Once a fault is identified, the decision logic switches its control to the safety controller and tries to recover the HP platform by restarting the system.

Table II shows the observations by the decision logic and the corresponding faults at the HP 


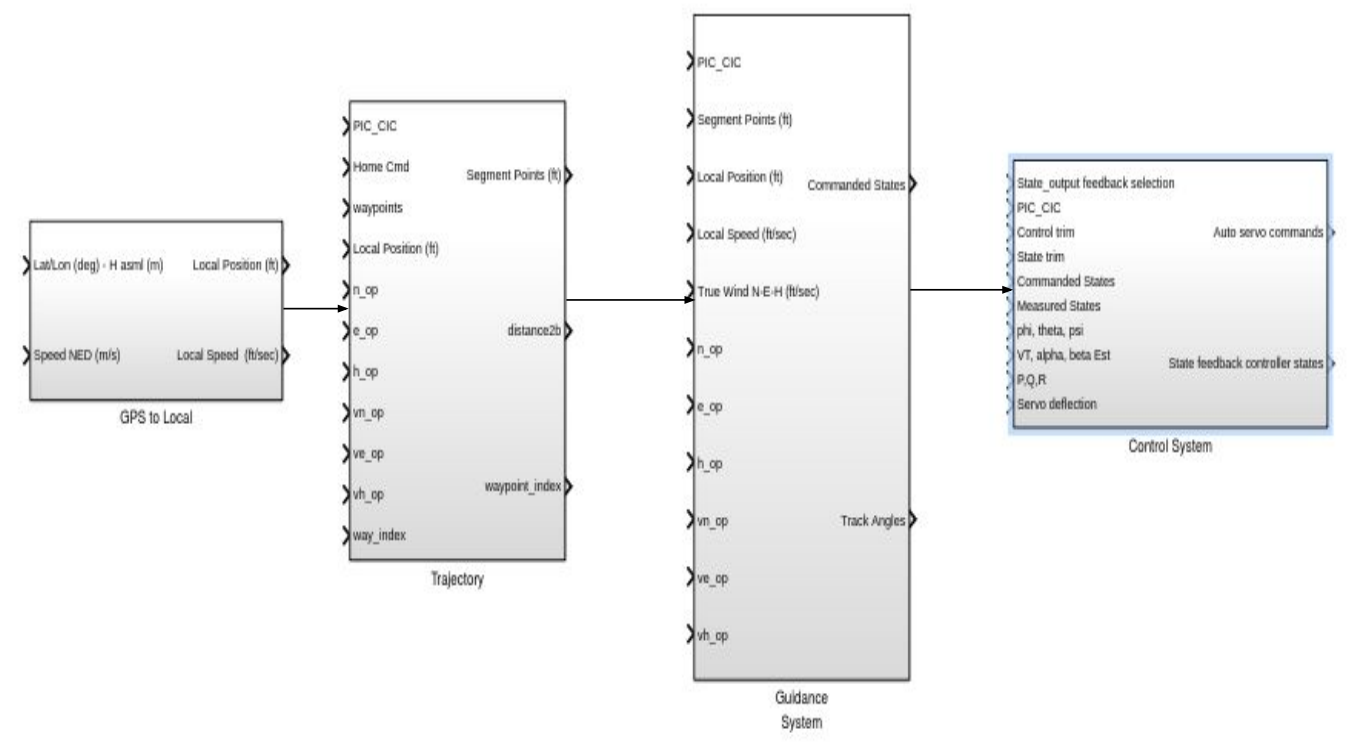

Figure 4.3: Autonomous controller block diagram 


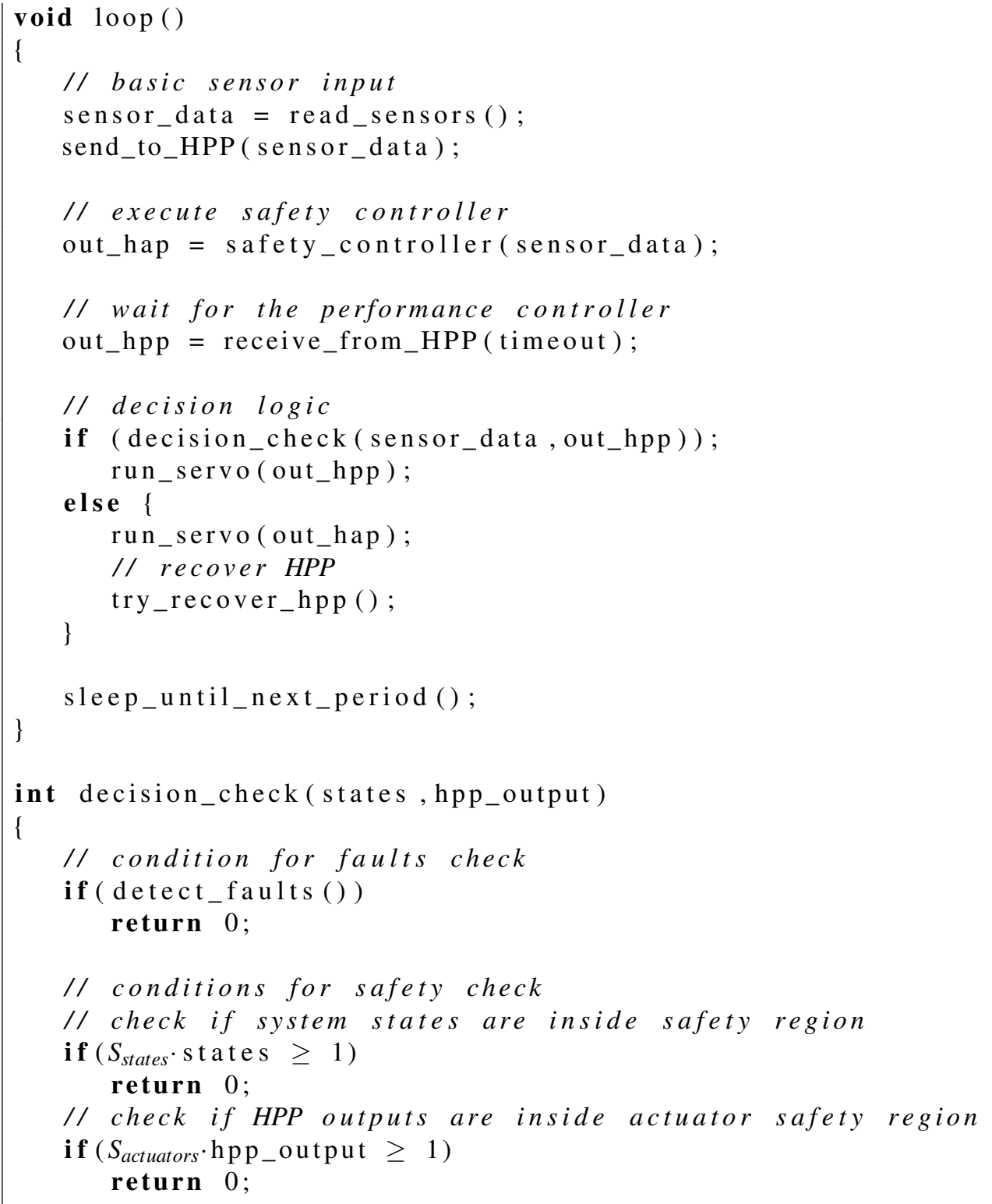

Figure 4.4: Decision logic on Arduino. 
platform. First, 'No output' means that the decision logic does not receive performance controller's control outputs. This can be caused by software faults such as OS or controller crashes. In our current implementation, the decision logic waits one more control period before it makes a switch to the safety controller. If the outputs are received in the next period, the decision logic's observation is 'Delayed output' and the system continues to use the performance controller. The aircraft states for every cycle, must be inside a safety region in order to maintain the stability of the system.

\subsubsection{Safety Region}

The safety region depends on the aircraft dynamics and hence this region varies from one aircraft to another. The safety region for the UAV is defined based on Section 3.2. We added our custom built avionics whose design is based on our UAV simplex architecture to a glider named DG808 that is shown in Figure 1.1. The safety region for this UAV is defined based on its states and the inputs to the system.

The critical angle of attack for the DG808 glider is $30 \mathrm{deg}{ }^{1}$. Therefore the safety region containing the set of states where UAV will not have stall condition is given by,

$$
S=\left(\begin{array}{ccccccc}
0 & 1 / 30 & 0 & 0 & 0 & 0 & 0 \\
0 & 0 & 1 / 30 & 0 & 0 & 0 & 0
\end{array}\right)\left(\begin{array}{c}
\text { airspeed } \\
\text { angleofattack } \\
\text { sideslipangle } \\
\text { roll } \\
\text { pitch } \\
P \\
Q \\
R
\end{array}\right) \leq\left(\begin{array}{c}
1 \\
1
\end{array}\right)
$$

The actuators used in this UAV have their operation angles. The servo motors used for aileron and rudder are capable of handling deflection angles from -15 to +15 deg where as the servo motor

\footnotetext{
${ }^{1}$ The critical angle of attack for DG808 was calculated using Advanced Aircraft Analysis software. [1].
} 
assigned to the elevator operates with in -20 to $+20 \mathrm{deg}$. Therefore the actuator safety region is given by,

$$
S_{u}=\left(\begin{array}{ccc}
1 / 15 & 0 & 0 \\
-1 / 15 & 0 & 0 \\
0 & 1 / 15 & 0 \\
0 & -1 / 15 & 0 \\
0 & 0 & 1 / 20 \\
0 & 0 & -1 / 20
\end{array}\right)\left(\begin{array}{c}
\text { aileron } \\
\text { rudder } \\
\text { elevator }
\end{array}\right) \leq\left(\begin{array}{c}
1 \\
1 \\
1 \\
1 \\
1 \\
1
\end{array}\right)
$$

\subsubsection{Decision Logic Conditions}

In this section we define the decision logic conditions for DG808 glider. In every cycle, the outputs of high performance platform are sent to the decision logic and evaluated against all the conditions mentioned in this section. If the controller output is valid against all these conditions then the decision logic says that high performance platform is not safe and allows it to handle the system. The conditions are described using Linear Temporal Logic (LTL) which is a mathematical language for describing linear time properties [29].

\subsubsection{Condition 1: Valid Controller Output}

- Aim : To check whether the controller generates the outputs within the servo motor range. The controller may diverge and produce outputs that cannot be handled by the servo motors or the controller program might have crashed.

- LTL condition :

$$
\begin{aligned}
& -15 \text { deg } \leq \square \text { aileron } \leq 15 \text { deg } \\
& -20 \text { deg } \leq \square \text { elevator } \leq 20 \text { deg }
\end{aligned}
$$




$$
-15 \mathrm{deg} \leq \square \text { rudder } \leq 15 \mathrm{deg}
$$

- Explanation : The controller generally produces aileron, elevator and rudder surface outputs. These outputs are given to the servo motors. The servo motors have operation range. This condition is used to verify whether the controller produces outputs within the operation range or not.

\subsubsection{Condition 2: Kalman Filter Divergence}

- Aim : Most of the autonomous system will use kalman filters in their logic to estimate some of the missing sensor values or to reduce the noise level in the sensor data. These kalman filter, if designed with improper gains and coefficients, are highly unstable and will diverge the controller.

- LTL condition : The controller program in our test bed estimates the side slip and angle of attack.

$$
-30 \mathrm{deg} \leq \square \alpha \leq 30 \mathrm{deg}^{2}
$$

Where $\alpha$ is the angle of attack

$$
-30 \mathrm{deg} \leq \square \beta \leq 30 \mathrm{deg}^{2}
$$

where $\beta$ is the side slip angle

- Explanation : The side slip and angle of attack estimation should always be converged. If this condition fails then the kalman filter has diverged. The failure of this condition is due to improper value for the filter gains and co efficient. This failure will eventually lead to failure of condition in the section 4.3.2.1.

\footnotetext{
${ }^{2}$ This value is calculated using AAA software [1] based on the vehicle dynamics.
} 


\subsubsection{Condition 3: Sensor Communication with the Performance Controller}

- Aim : In UAV simplex architecture, the performance controller is not directly connected to the sensors. All the sensors are connected to the assurance platform and then passed to the performance controller through a communication medium. In our glider UAV, we used the USB communication between the platforms. Unfortunately, the USB communication is not always reliable. The connection can be lost due to some vibrations to the system and it can restored by re-spawning the program. In order to restore the communication, we currently treat this as an safety issue and restart the performance platform.

- LTL condition : The high performance platform always monitors the USB health condition and sends this health status to the decision logic in high assurance platform.

$$
U S B \models T R U E
$$

where $U S B$ denotes the USB health status

- Explanation : The communication between high performance platform and the sensors is always monitored by the high performance platform and this health condition should always be good for the high performance controller. This failure will eventually lead to failure of condition in the sections 4.3.2.1 and 4.3.2.2.

\subsubsection{Condition 4: Stall Speed Condition}

- Aim : An aircraft will experience a stall condition when its angle of attack is greater than threshold value.

- LTL condition :

$$
\left((\text { alt }>\text { alt } 0) \rightarrow\left(v_{\text {air }}>v_{\text {stall }}\right)\right)
$$

where alt is the altitude measured and alt $t_{0}$ is the ground altitude, $v_{\text {air }}$ is the air speed measured by the sensor and $v_{\text {stall }}$ is the stall speed of the aircraft and its value depends on the 
flight dynamics.

- Explanation : In most of the small commercial UAVs, there is no dedicated sensor for measuring this angle. But the stall condition can be checked using the altitude and air speed measured from GPS sensor and the pitot tube respectively. The condition given in Equation 4.9 shows that when the aircraft is air bound identified by monitoring the altitude value from GPS sensor, then the air speed of the vehicle should be always greater than the stall speed. If this condition fails, then it indicates that the aircraft is climbing up at a high angle such that the pitot tube is not able read the air speed.

\subsubsection{Condition 5: FAA Regulation on Altitude}

- Aim : The Federal Aviation Administration (FAA) has regulations like the commercial UAV should always fly within 400 feet [3] from ground. This condition checks whether the high performance controller is obeying this regulation.

- LTL condition :

$$
\square(\text { alt } \leq 400)
$$

- Explanation : When the high performance controller produces output that makes the aircraft to climb altitude above 400 feet from ground level, then this condition fails and the decision logic switches to high assurance controller and restarts the high performance controller. 


\section{Chapter 5}

\section{Evaluation}

In this chapter, we evaluate our UAV simplex architecture through a case study using Hardwarein-the-Loop (HiL) simulation. We also talk about the flight test using our custom built avionics. More detailed explanation regarding the flight test is described in Section 5.2.

\subsection{HiL Test}

Hardware-in-the-Loop (HiL) simulation is a technique that is used for testing control systems. In UAVs the physical parts (sensor and actuators) are connected with the cyber part(controller). In HiL testing the physical parts are replaced by simulation(flight simulator) that can mimic the sensors and actuators of the UAV.

\subsubsection{Setup}

Figure 5.1 shows the Hardware-in-the-Loop (HIL) experiment setup that we used to evaluate our Simplex based UAV system. The experiment setup consists of an Arduino Due, a Tegra TK1, and a laptop. The Arduino Due executes the safety controller and decision logic, while the Tegra TK1 platform executes the performance controller. Together, they form the cyber system of the UAV. On the other hand, the laptop runs the ground station and a custom built flight simulator. The flight simulator, which is implemented in Matlab, provides simulated sensor values to both 
UDP COMMUNICATION

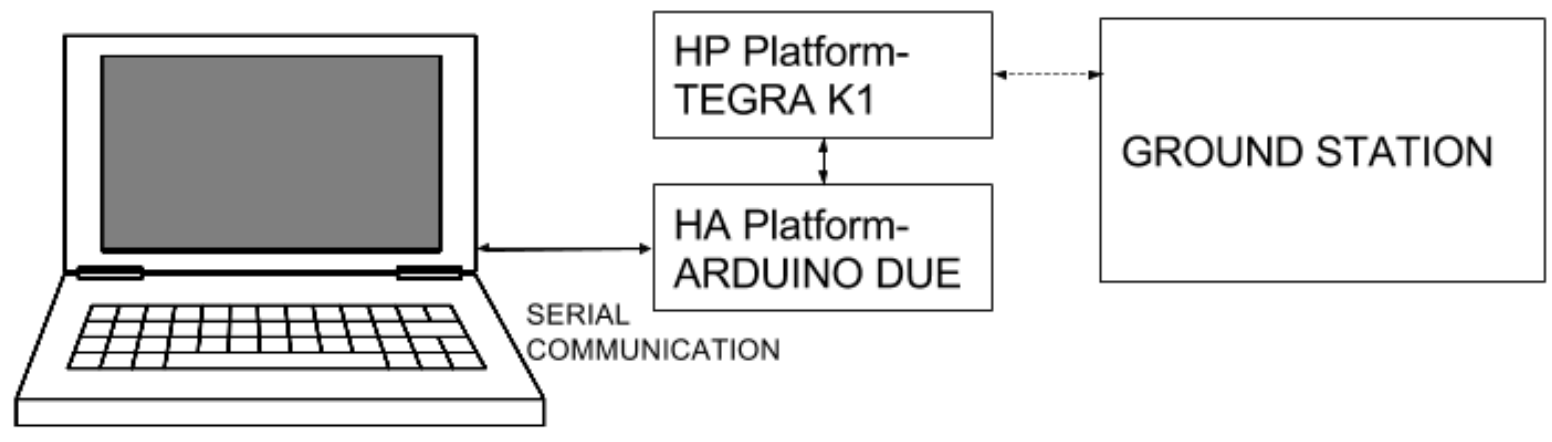

\section{SIMULATION FOR SENSORS AND SERVOS}

Figure 5.1: Experiment setup for UAV simplex

the controllers and in turn receives the control command surface outputs from the controllers and calculate the next inputs.

\subsubsection{Case Study: Performance Controller Crash}

In this case-study, we intentionally crash the performance controller in the HP platform to evaluate the system's ability to detect and recover from the fault.

Figure 5.3 shows the outputs of the performance controller (top), the safety controller (middle), and the decision logic (bottom), collected over 200 seconds duration (Note that the figure shows only one of the four control outputs.) For the first 100 seconds, both safety and performance controllers are working in parallel on HA and HP platforms, respectively. In this normal operation mode, the decision logic chooses to use the outputs of the performance controller. At 100 seconds, however, we inject a fault by manually terminating the performance controller in the HP platform. Because the decision logic observes no outputs from the performance controller, it switches to use the outputs from the safety controller. At about 130 seconds, the performance controller is restarted and produces control outputs. However, the decision logic does not immediately switch back to the performance controller because the outputs of the performance controller is not stabilized yet. The 


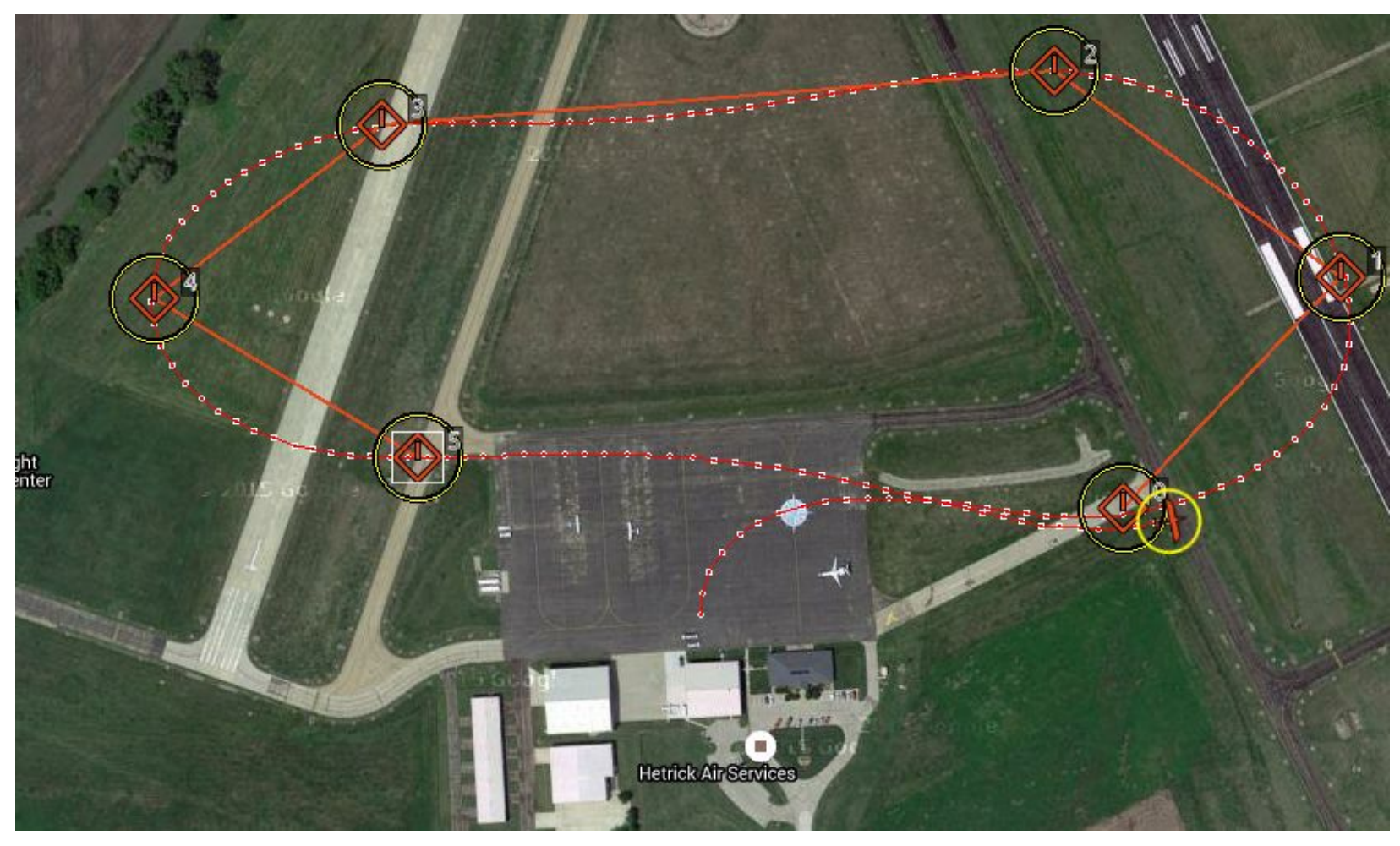

Figure 5.2: The flight path 

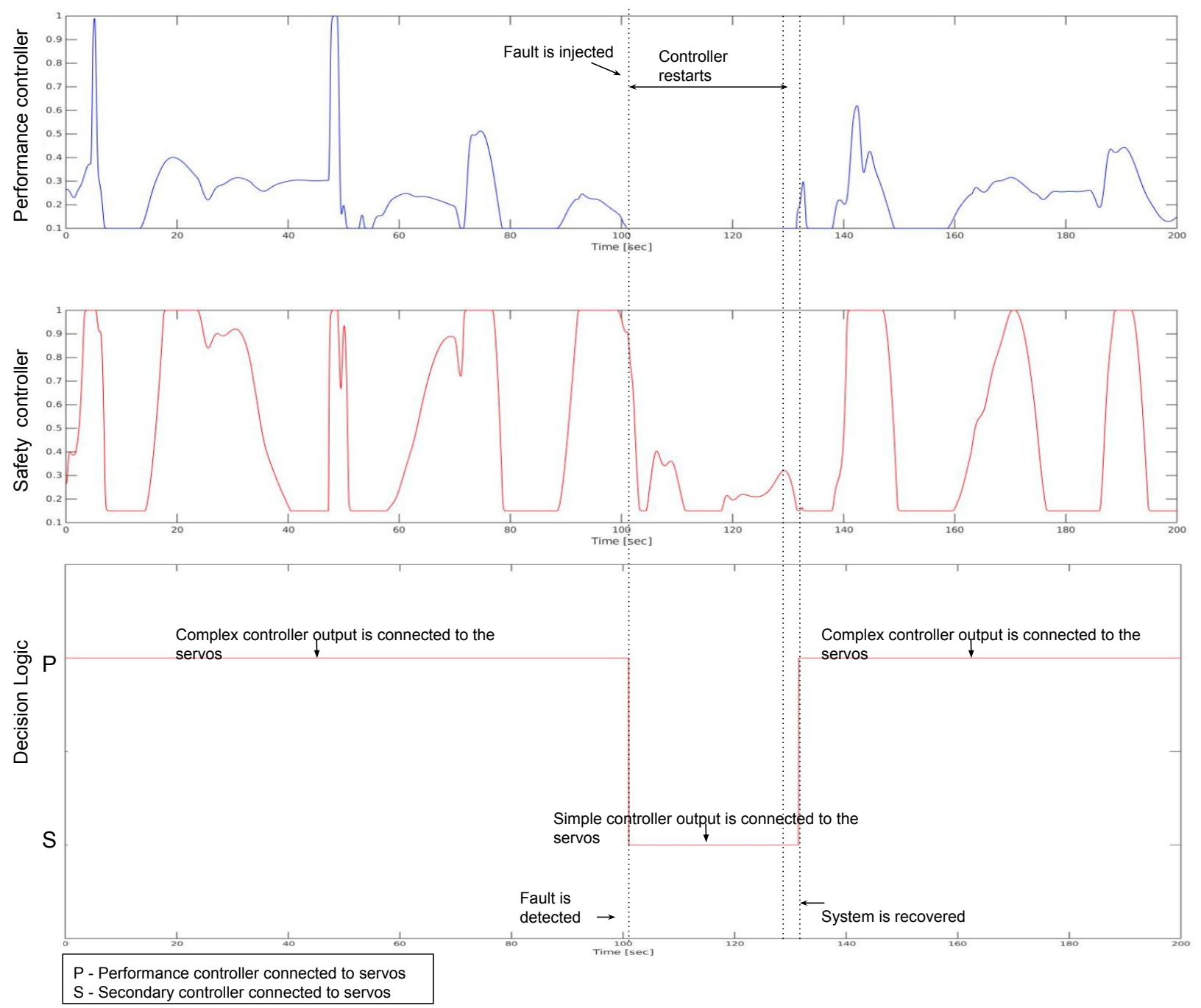

Figure 5.3: Outputs of safety controller, performance controller, and decision logic. A fault is injected at time 100 second.

stability factor depends on the convergence of the filters and the guidance logic of the controller. Once the outputs are stabilized, the decision logic switches back to use performance controller's outputs. Figure 5.2 shows the ground station tracking of the UAV system during the test. The solid orange lines denote the waypoint trajectory uploaded to the UAV system from the ground station and the red lines show the trajectory followed by the flight and the white dots represent the position update from the UAV system on the ground station. Our UAV system is designed to update the ground station at $10 \mathrm{~Hz}$. 


\subsection{Flight Test}

In this section, we discuss about the test that we conducted using our avionics (mentioned in Chapter 4) in a real aircraft shown in Figure 1.1. In this test, we disabled the safety controller and decision logic in high assurance platform. Instead, we used two performance controllers in high performance platform and we manually switched the control from one performance controller to another. The aim of this test was to ensure that the aircraft is stable, while switching the control from one controller to another.

The test procedure was to takeoff the aircraft using pilot commanded RC values. Once the aircraft reached a steady state, the autopilot was engaged. The way points required by the autopilot was uploaded from the ground through ground station application. We integrated two autonomous controller programs ("autopilot1 and autopilot2") in the high performance platform of our avionics. Both the controllers mission was to follow the waypoints uploaded from ground station. Instead of using decision logic to switch control from one controller program to another controller, the ground station application was used for switching. We modified our avionics program to accept the switching command from ground station to switch between the controllers. It can be seen from the third sub plot in Figures 5.4, 5.5, 5.6 and 5.7, the control was switched from one controller to another. The sub plot shows us that at $270^{\text {th }}$ second of the test, value in this plot has changed from ' 0 ' to ' 1 ' indicating that the avionics has switched the control from "autopilot 1 " controller to "autopilot2" controller. The control was again switched back to "autopilot1" controller at $350^{\text {th }}$ second of the flight test. The switching between controllers was repeated in the shorter and longer leg of the mission trajectory to see any sort of instability or rough transition.

We have recorded the video of ground station during the test. The details about this video is mentioned in Apendix A.

Figures 5.4, 5.5, 5.6 and 5.7 shows the states of the system while it was in autopilot. The $\mathrm{x}$-axis of the figures shows the time in seconds and the y-axis shows the angles in deg and rates in deg/s respectively. It can be seen from the figures that there was no sudden spike in any states during the switch between controllers (at $270^{\text {th }}, 350^{\text {th }}$ and $380^{\text {th }}$ second of the flight test) and there was no 

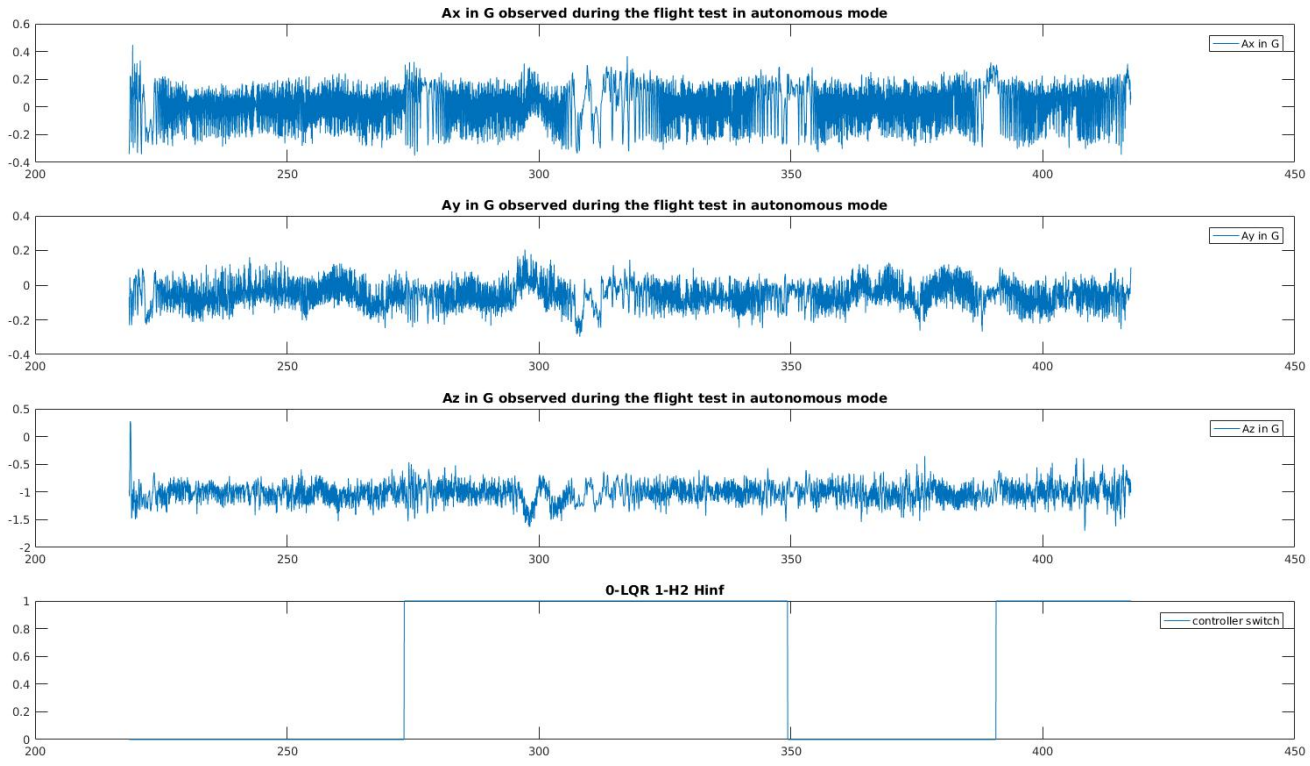

Figure 5.4: Accelerator values measured from flight test, X-axis: Time in seconds, Y-axis: Acceleration in $\mathrm{G}$
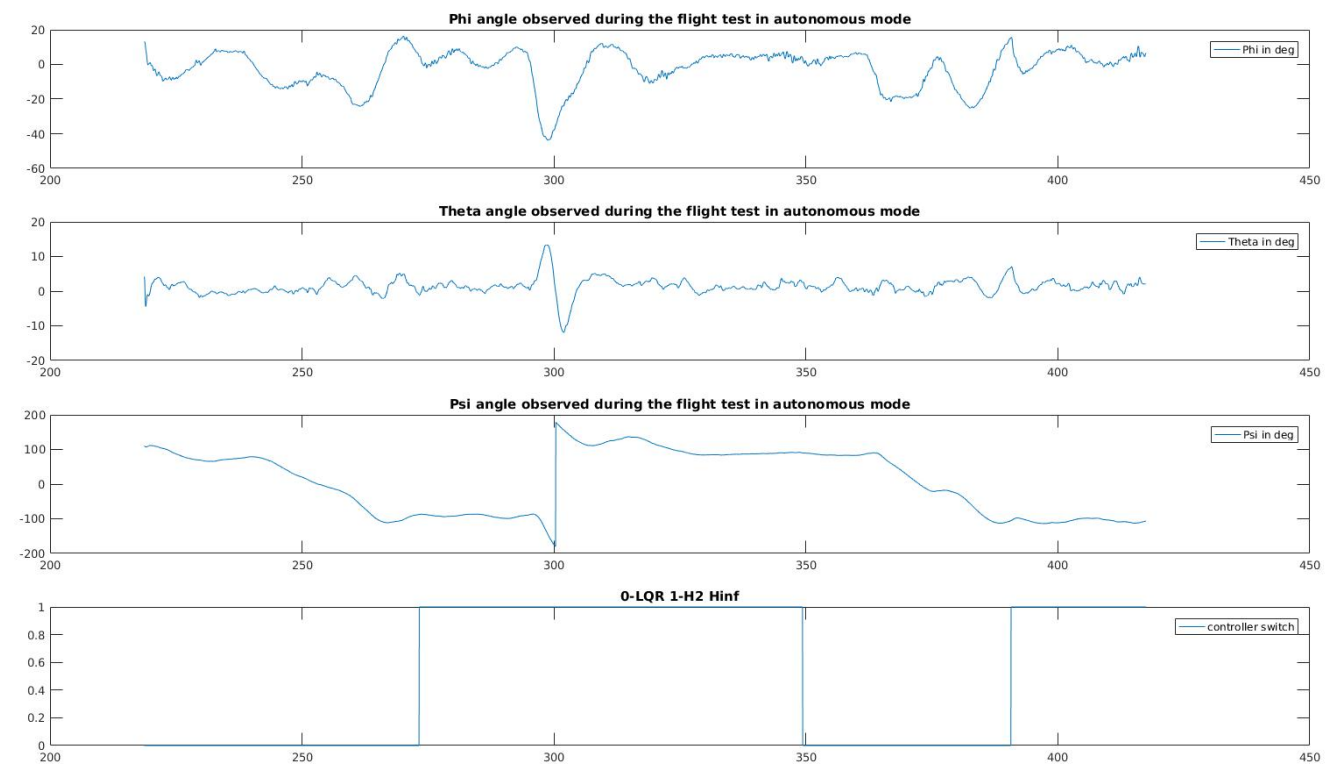

Figure 5.5: Aircraft orientation in Euler angles measured from flight test, X-axis: Time in seconds, Y-axis: Euler angles in degrees 

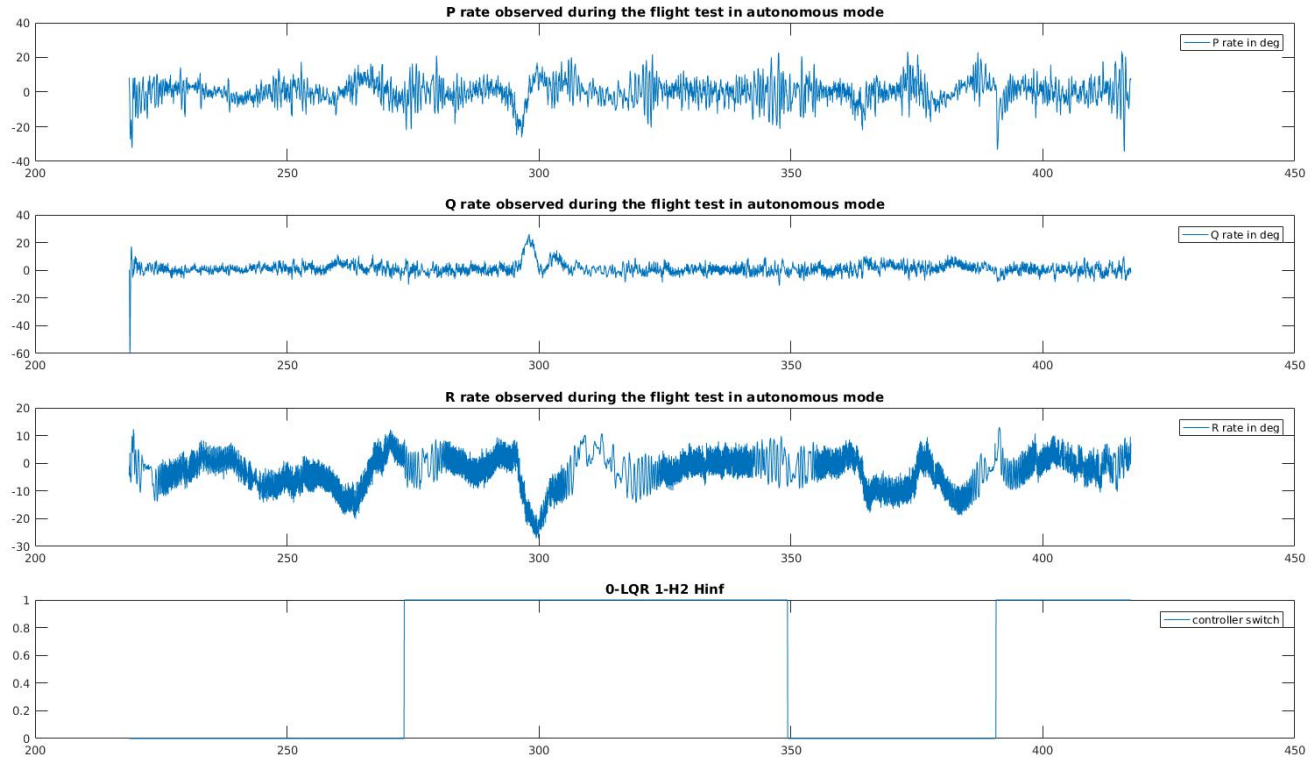

Figure 5.6: Body rates measured from flight test, X-axis: Time in seconds, Y-axis: Body rates in $\operatorname{deg} / \mathrm{s}$
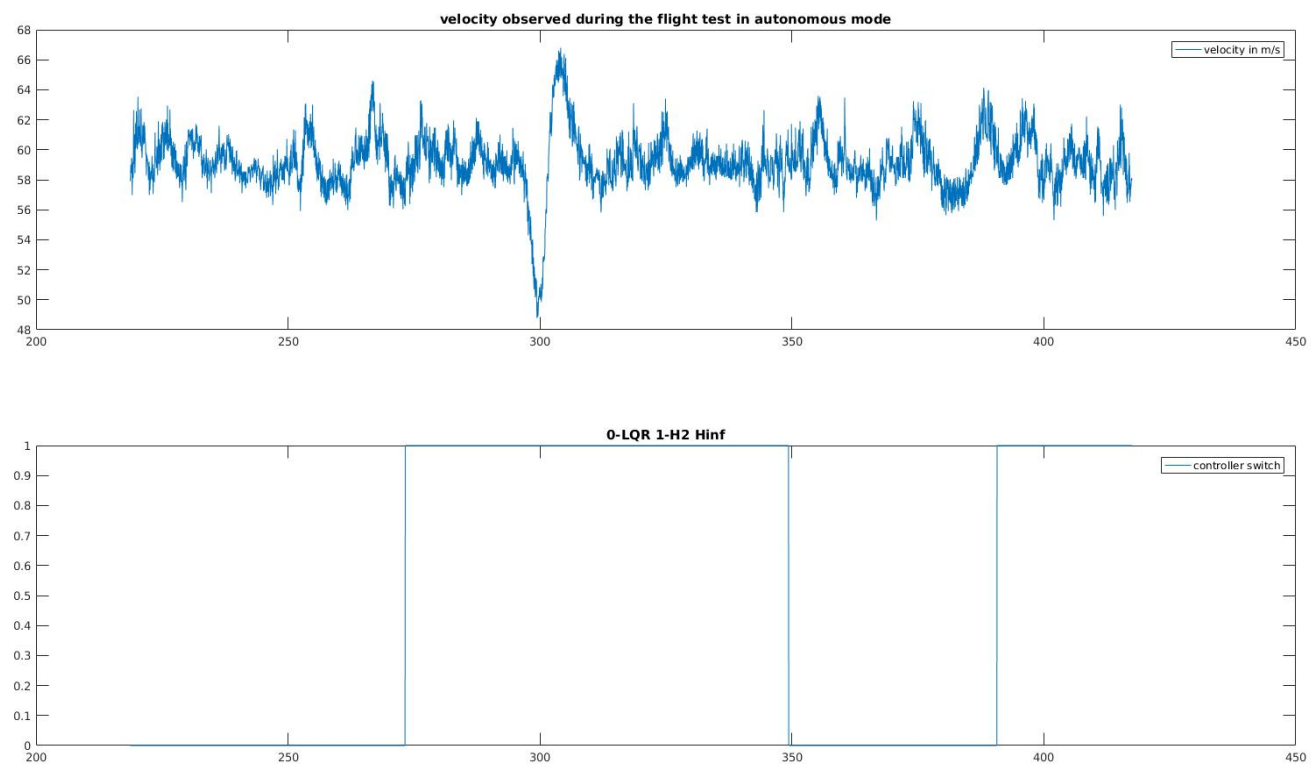

Figure 5.7: Aircraft velocity measured from flight test, X-axis: Time in seconds, Y-axis: velocity in $\mathrm{ft} / \mathrm{s}$ 


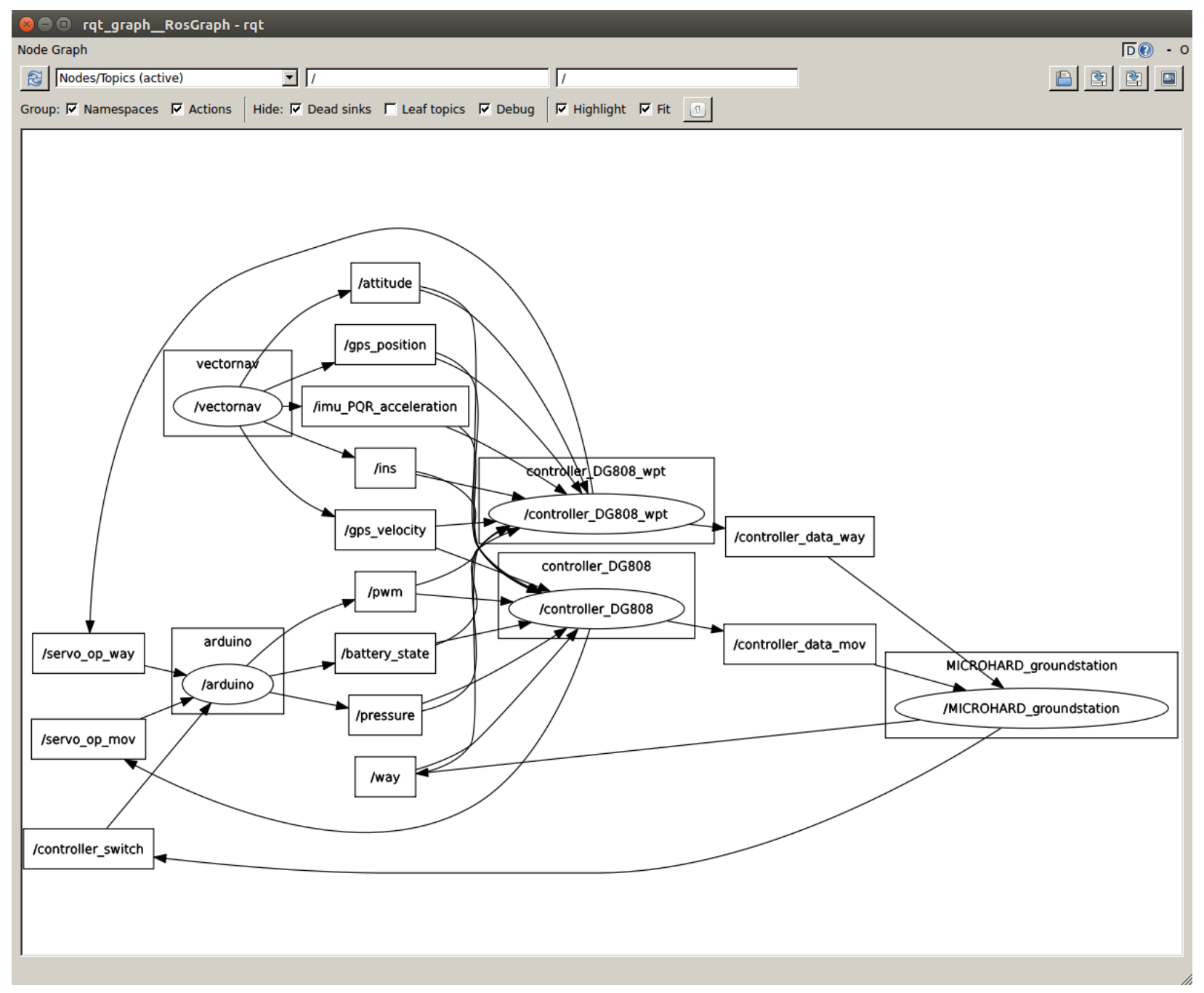

Figure 5.8: Rosgraph plot showing the nodes and topics and for the switch between controllers case 
instability in the system behavior, thus the switching between the controllers in the middle of the mission was smooth while following the mission.

However, the above test did not include the decision module for fault detection because the aim of the test was to see the stability of the aircraft, after switching the controller. In this test, the switch between the controllers was initiated by the ground station operator. Figure 5.8 shows the ROS software architecture of the test. As a future work, we plan to integrate the decision logic and do a flight test with fault injection. 


\section{Chapter 6}

\section{COTS Autopilot with UAV Simplex}

\section{Architecture}

In UAV simplex architecture, two different hardware platforms were used. They were classified as High Performance platform and High Assurance platform. So far, we have used an ARM CortexM3 based custom built computing board as the HA platform, which runs the decision logic and the safety controller.

The realization of UAV simplex architecture was hindered by this custom built board since it takes more time and cost to develop and verify. Hence in this chapter, we replaced the custom built board with an available COTS autopilot that also provides sensor acquisition and a simple mission controller. We used Pixhawk [5] as our COTS autopilot along with our onboard computer.

Pixhawk, as shown in Figure 6.1, is a famous COTS autpilot that also provides easy interface to connect with an on board computer and runs a simple PID controller that follows the uploaded mission from ground station. Pixhawk uses a cortex-M4F processor that operates at $168 \mathrm{MHz}$. It also provides 14 PWM pins, that are required for the servo motors and supports peripherals like I2C and CAN, that are essential for communicating with the sensor. However, the main advantage of using Pixhawk over our custom built avionics is the that, the Pixhawk software framework provides integration support to the sensors required for an UAV. Whereas, in our custom built avionics, we 


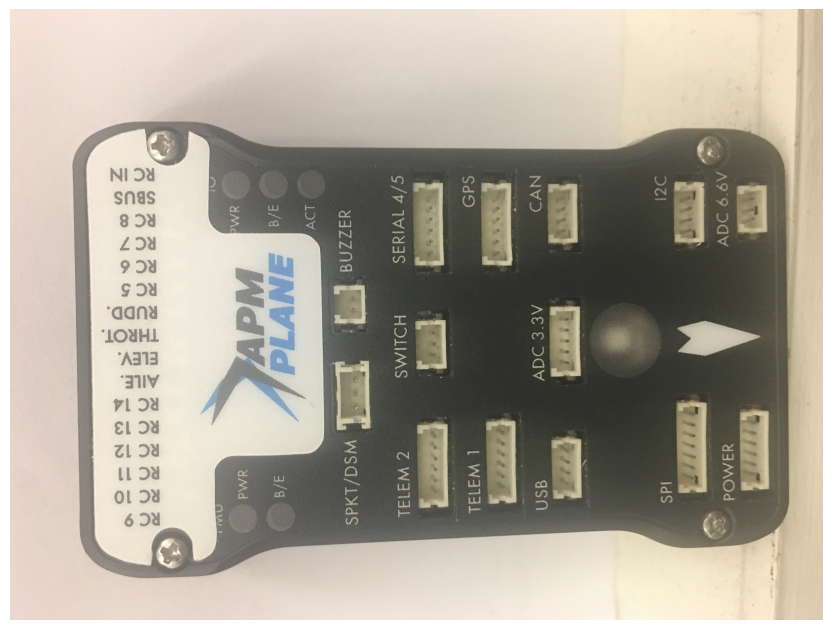

Figure 6.1: Pixhawk outer view

need to take care of sensor integration.

\subsection{Implementation}

The following steps are involved in replacing the custom built avionics board with the Pixhawk COTS board.

The pixhawk board comes with two telemetry ports. These ports output all the sensor data encrypted using mavlink protocol. The telemetry port 1 will be always used for communication with ground station. Hence the communication between pixhawk and the onboard computer is established using a usb2ttl converter between telemetry port 2 and the onboard computer.

The mavlink encrypted data is available to the onboard computer. These data should be decrypted before sending them to the complex controller algorithm. The ROS provides a mavlink encryption and decryption package called MAVROS. MAVROS decrypts these data and publishes sensor data. To support our complex controller, an extra ROS node is implemented that converts the sensor data from MAVROS topic to the topics supported by the complex controller node.

The Figure 6.2 shows the rosgraph containing all the nodes and topics in this setup. Once the inputs are given to the on board controller algorithm, the output will be generated. The pixhawk also generates its output value. The values generated by the pixhawk should be replaced with the 


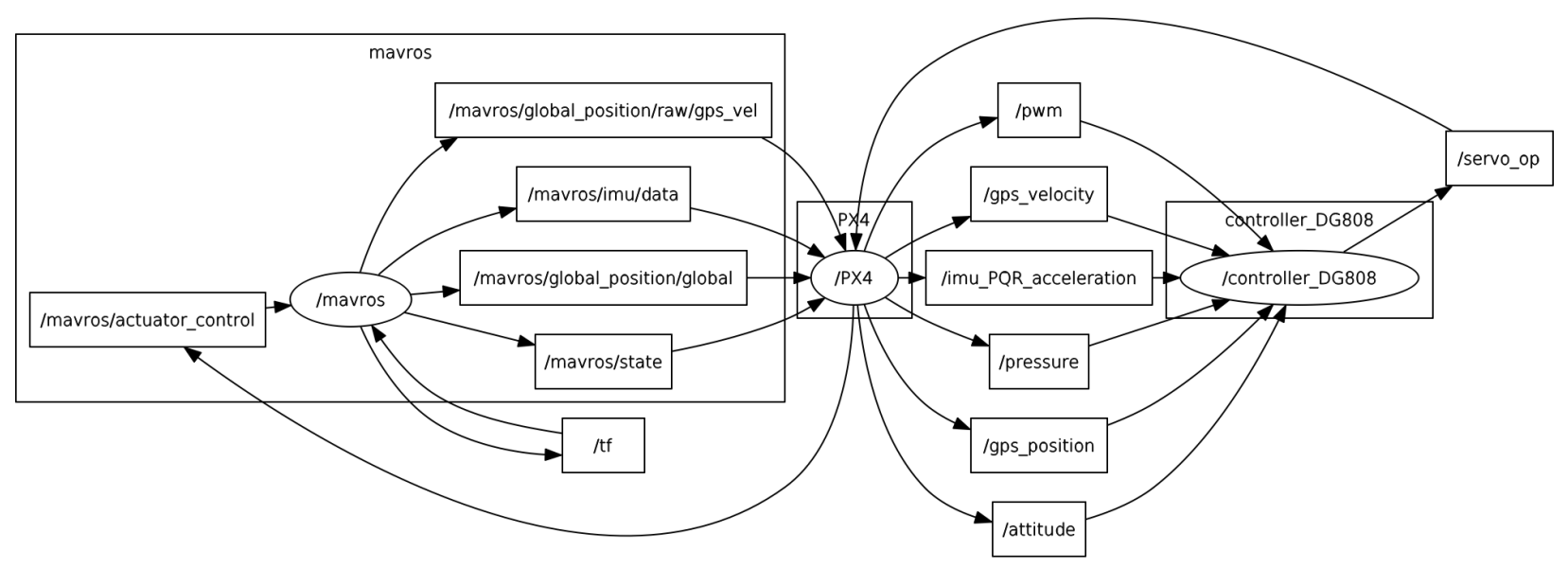

Figure 6.2: ROS nodes outline for PX4 and the controller program

values from the on board computer. For this, Pixhawk supports the offboard mode that rewrites its output with the on board computer's output.

\subsection{HiL Test}

The UAV simplex architecture using the Pixhawk is in Hardware In the Loop simulation. The setup consists of the following applications.

1. Xplane Flight gear simulator: Xplane [9] simulator runs the model of the aircraft and it takes the controller output as its input and generates the states. These states are sent to the controller program.

2. QGround station: The states generated by the Xplane are delivered to the controller program running in the pixhawk through Qground station. The Qground station is connected with the Pixhawk and communicates with the Xplane using UDP communication.

The Qground station receives all the state information from Xplane. Then, Qground station encrypts the values using mavlink protocol. The mavlink encrypted values are given to the on board computer. The on board computer generates the control output based on the state information and sent them to Xplane through Qground station. 


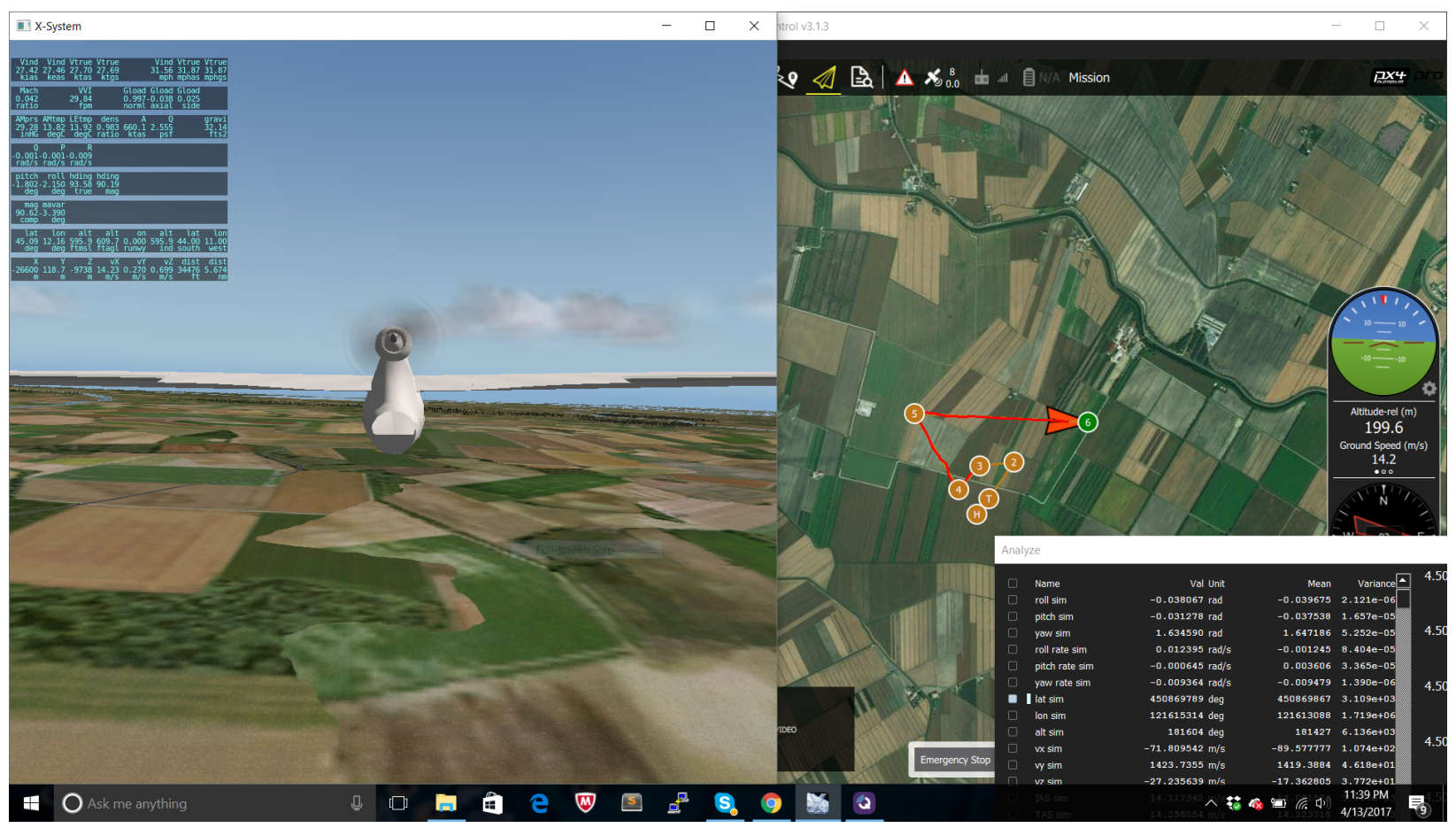

Figure 6.3: Hardware in the Loop for PX4 as the safety platform

In this work, we have not added the decision logic into the Pixhawk. The Pixhawk will automatically switch its mode from "offboard" to "return to land" when it stops receiving the values from on board computer. This feature provides safety against the fail stop case. But it cannot detect and recover from the unsafe output conditions. Adding the decision logic into the Pixhawk is our future work.

\subsection{Aircraft Setup using Pixhawk and On Board Computer}

We have installed the avionics containing Pixhawk (as a HA platform) and an Odroid-XU4 board (as a HP platform). As shown in the Figure 6.4, this is the avionics setup installed in the aircraft named Skyhunter. 


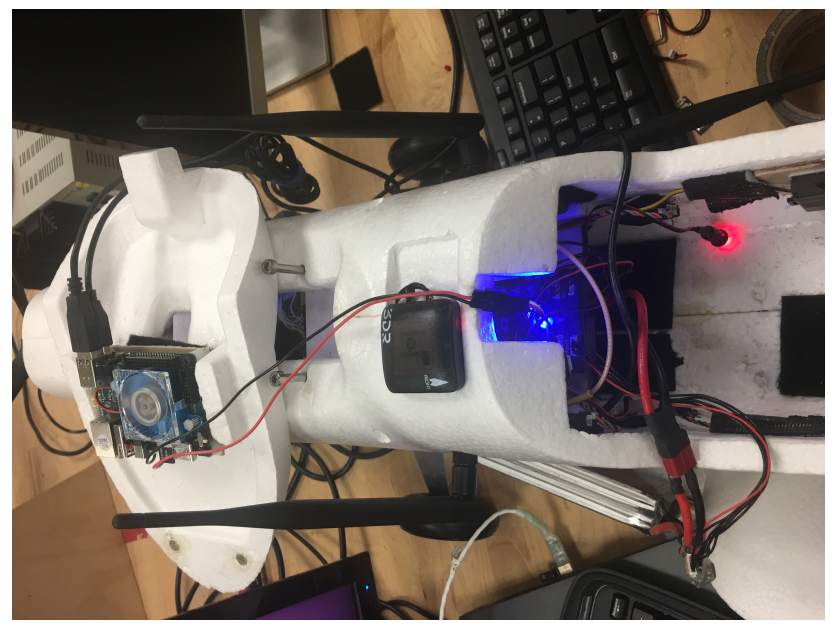

Figure 6.4: Skyhunter using Pixhawk and odroid avionics 


\section{Chapter 7}

\section{Conclusion}

We have presented a fault-tolerant UAV design based on the Simplex [35] architecture. The proposed design uses two heterogeneous platforms with distinct reliability and performance characteristics.

Our main idea is that we use a reliable but less performance hardware platform, we call a High-Assurance (HA) platform, to be responsible for safety while we use a more performance, but potentially less reliable platform, which we call a High- Performance (HP) platform, for performance and intelligence of the UAV. During the normal operation, the HP platform is responsible for controlling the UAV. However, if it fails due to transient hardware faults or software bugs, the HA platform will immediately take over until the HP platform recovers. As such, our design provides a much needed fail-operational property in the UAV.

We have implemented the proposed design on an actual UAV using a low-cost Arduino and a high-performance Tegra TK1 platform. We have demonstrated the system's ability to detect and recover from failures through a set of experiments in a hardware-in-the-loop simulation setting. We did a flight test to show that the system is stable after switching between the controllers. We have also showed using hardware in the loop simulation that UAV simplex architecture can be implemented using COTS autopilot. In the future, we plan to implement more sophisticated vision sense-and-avoid capability. 


\section{References}

[1] Advanced Aircraft Analysis. http://www.darcorp.com/Software/AAA/.

[2] Aircraft stall. http://www. controlchat.com/physics-of-flight-the-stall/.

[3] FAA Increases Altitude Limit for Commercial UAVs. https://www aiaa.org/Detail. aspx?id=33070/.

[4] Microhard. http://www.microhardcorp.com/n920.php.

[5] Pixhawk COTS Autopilot. https://pixhawk.org/.

[6] Qground station. http://qgroundcontrol.org/.

[7] Simulink. http://www.mathworks.com/products/simulink/.

[8] Vn-220 rugged gps/ins. http://www.vectornav.com/products/vn200-rugged/.

[9] Xpalne flight simulator. http://www.x-plane.com/.

[10] F. A. T. Abad, R. Mancuso, S. Bak, O. Dantsker, and M. Caccamo. Reset-based recovery for real-time cyber-physical systems with temporal safety constraints. In 2016 IEEE 21 st International Conference on Emerging Technologies and Factory Automation (ETFA), pages 1-8, Sept 2016.

[11] F. Abdi, M. Hasan, S. Mohan, D. Agarwal, and M. Caccamo. Resecure: a restart-based security protocol for tightly actuated hard real-time systems. In 1st Workshop on Security and Dependability of Critical Embedded Real-Time Systems (2016). 
[12] F. Abdi, R. Tabish, M. Rungger, M. Zamani, and M. Caccamo. Application and system-level software fault tolerance through full system restarts. In Proceedings of the 8th International Conference on Cyber-Physical Systems, ICCPS '17, pages 197-206, New York, NY, USA, 2017. ACM.

[13] Aeronautical Radio Inc. Avionics Application Standard Software Interface (ARINC) 653, 2013.

[14] S. Bak, D. Chivukula, O. Adekunle, M. Sun, M. Caccamo, and L. Sha. The system-level simplex architecture for improved real-time embedded system safety. In Real-Time and Embedded Technology and Applications Symposium (RTAS), pages 99-107. IEEE, 2009.

[15] R. Baumann. Soft errors in advanced computer systems. Design \& Test of Computers, IEEE, 22(3):258-266, 2005.

[16] J. Bengtsson, K. Larsen, F. Larsson, P. Pettersson, and W. Yi. UPPAAL: a tool suite for automatic verification of real-time systems. Springer, 1996.

[17] P. Bieber, F. Boniol, M. Boyer, E. Noulard, and C. Pagetti. New Challenges for Future Avionic Architectures. AerospaceLab Journal, (4), 2012.

[18] S. Borkar. Designing reliable systems from unreliable components: the challenges of transistor variability and degradation. Micro, IEEE, 25(6):10-16, 2005.

[19] D. Brière and P. Traverse. AIRBUS A320/A330/A340 electrical flight controls-A family of fault-tolerant systems. In Fault-Tolerant Computing, pages 616-623. IEEE, 1993.

[20] J. B. Dabney and T. L. Harman. Mastering simulink. Pearson/Prentice Hall, 2004.

[21] P. Fritzson. Principles of object-oriented modeling and simulation with Modelica 2.1. John Wiley \& Sons, 2010.

[22] G. A. Garcia, S. Keshmiri, and R. Colgren. Advanced h-infinity trainer autopilot. AIAA Modeling and Simulation Technologies Conference, August 2010. 
[23] M. Hoffmann, F. Lukas, C. Dietrich, and D. Lohmann. dosek: the design and implementation of a dependability-oriented static embedded kernel. In Real-Time and Embedded Technology and Applications Symposium (RTAS), 2015 IEEE, pages 259-270. IEEE, 2015.

[24] G. Klein, K. Elphinstone, G. Heiser, J. Andronick, D. Cock, P. Derrin, D. Elkaduwe, K. Engelhardt, R. Kolanski, M. Norrish, et al. sel4: Formal verification of an os kernel. In Symposium on Operating Systems Principles (SOSP), pages 207-220. ACM, 2009.

[25] O. Kotaba, J. Nowotsch, M. Paulitsch, S. Petters, and H. Theilingx. Multicore in realtime systems temporal isolation challenges due to shared resources. In Industry-Driven Approaches for Cost-effective Certification of Safety-Critical, Mixed-Criticality Systems, 2013.

[26] R. E. Lyons and W. Vanderkulk. The use of triple-modular redundancy to improve computer reliability. IBM Journal of Research and Development, 6(2):200-209, 1962.

[27] T. C. May and M. H. Woods. Alpha-particle-induced soft errors in dynamic memories. IEEE Transactions on Electron Devices, 26(1):2-9, Jan 1979.

[28] Y. Moy, E. Ledinot, H. Delseny, V. Wiels, and B. Monate. Testing or formal verification: Do-178c alternatives and industrial experience. Software, IEEE, 30(3):50-57, 2013.

[29] R. Murray. Lecture on linear temporal logic. http://www.cds.caltech.edu/ murray/ courses/afrl-sp12/L3_ltl-24Apr12.pdf, 2012.

[30] J. Nowotsch and M. Paulitsch. Leveraging multi-core computing architectures in avionics. In Dependable Computing Conference (EDCC), pages 132-143. IEEE, 2012.

[31] NVIDIA. NVIDIA Tegra K1 Mobile Processor, Technical Reference Manual Rev-01p, 2014.

[32] M. Quigley, K. Conley, B. Gerkey, J. Faust, T. Foote, J. Leibs, R. Wheeler, and A. Y. Ng. Ros: an open-source robot operating system. In ICRA workshop on open source software, volume 3, page 5, 2009. 
[33] RTCA. DO-178C Software Considerations in Airborne Systems and Equipment Certification, 2011.

[34] I. Sadeghzadeh and Y. Zhang. A review on fault-tolerant control for unmanned aerial vehicles (uavs).Infotech@Aerospace, 2011.

[35] L. Sha. Using simplicity to control complexity. IEEE Software, 18(4):20-28, 2001.

[36] J. Song and G. Parmer. C'mon: a predictable monitoring infrastructure for system-level latent fault detection and recovery. In Real-Time and Embedded Technology and Applications Symposium (RTAS), 2015 IEEE, pages 247-258. IEEE, 2015.

[37] P. Vivekanandan. Source code for our avionics. https://github.com/tharunprasanth/ drone_ros.

[38] M. K. Yoon, S. Mohan, J. Choi, J. E. Kim, and L. Sha. Securecore: A multicore-based intrusion detection architecture for real-time embedded systems. In 2013 IEEE 19th RealTime and Embedded Technology and Applications Symposium (RTAS), pages 21-32, April 2013.

[39] J. F. Ziegler. Terrestrial cosmic rays. IBM journal of research and development, 40(1):19-39, 1996. 


\section{Appendix A}

\section{Videos}

We have made few videos showing our HiL test

1) The Hardware in the Loop simulation using our custom built avionics, showing fault tolerance against the crash fault video can be found in this link https://youtu . be/p6BT3UN8qJE

2) The video playback of the ground station during the real flight test with switch between controllers can be found in this link https://www.dropbox.com/s/qrctgrqe5k1dz2i/CTRL_ Switch_LQR2H2-2017-03-26_18-09-41.avi?dl=0 


\section{Appendix B}

\section{Issues Faced and Solved}

This appendix includes information about some of the issues that we faced and solved while integrating and using our avionics, matlab code generation and in bench test.

\section{B.1 Divide by Zero}

We are using kalman filters in our controller program for prediction and estimation of the system states. Divide by zero occured at the estimation of 'VT', the true velocity which is calculated using the pressure value from the 'pitot tube'. Pitot tube is connected to a differential pressure sensor. When the UAV system is static in ground, it reads zero pressure that results in zero velocity value. This zero velocity is given to the kalman filter block as seen in Figure B.1. This block outputs 'NaN' and will cause the controller to diverge. We fixed this issue by giving a small constant value to the velocity, when it reads zero in ground.

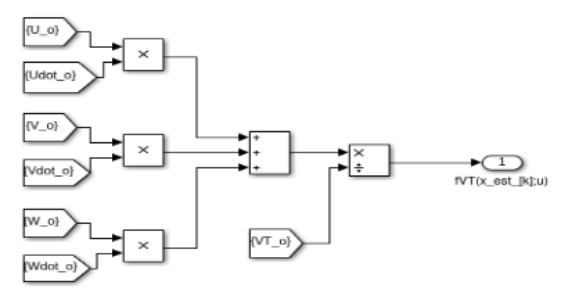

Figure B.1: Estimation of True velocity 


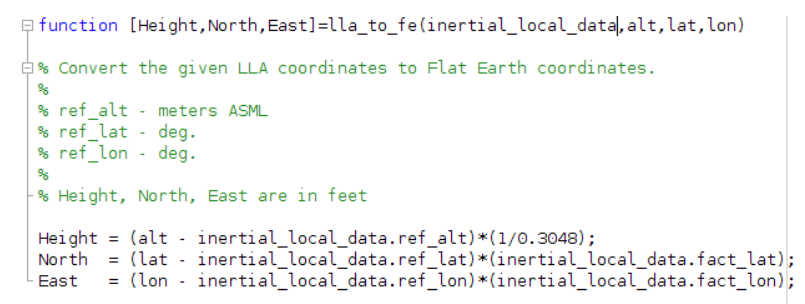

Figure B.2: Matlab code to convert GPS position from global to local

\section{B.2 Structure Ordering}

The autonomous controller in initial stages, is designed using Matlab. For the guidance block(guides in following the mission) in autonomous controller, the GPS position in local frame is required. Usually, all GPS sensors output GPS values in global frame. Hence we need to convert them from global to local frame. The Figure B.2 shows the Matlab code to convert the GPS position from global to local frame. The conversion consist of current GPS position in global frame, reference position in global frame and conversion factor based on the reference position. We use Matlab Embedded code generator to generate $\mathrm{C}$ code from the autonomous controller Matlab files. The Figure B.3 shows the generated C code. In generated code, the values of the conversion factor based on the reference position and the reference position in global frame are interchanged. This interchanged values lead to wrong guidance values. Hence the aircraft in hardware in the loop testing did not follow the mission. The reason for this value interchange in the generated code was, these variables are created as structures. The order in which the elements inside this structure was different from the order in which the elements inside the structure was defined. This declaration and definition mismatch showed no impact on Matlab simulation, but in code generation, this mismatch was reflected. This issue was resolved by following uniform structure order during its declaration and definition.

\section{B.3 USB Hub Power Issue}

In our avionics, we used usb connection to connect with our custom built data acquisition board, with GPS and IMU sensor and with the telemetry module to communicate with the ground station. 


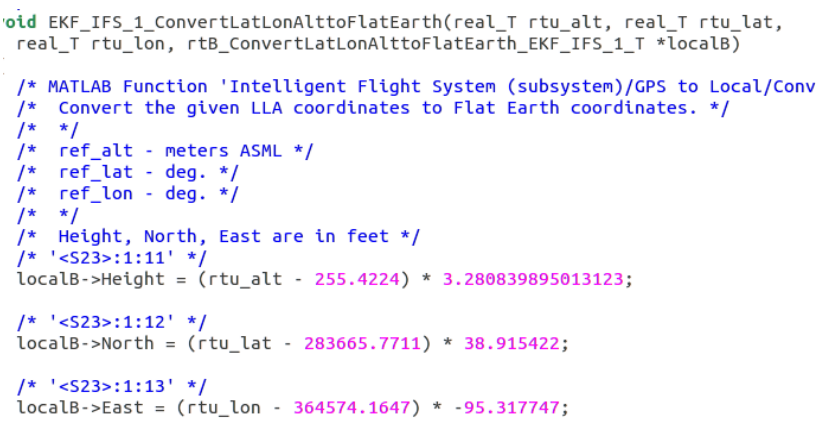

Figure B.3: Matlab generated C code to convert GPS position from global to local

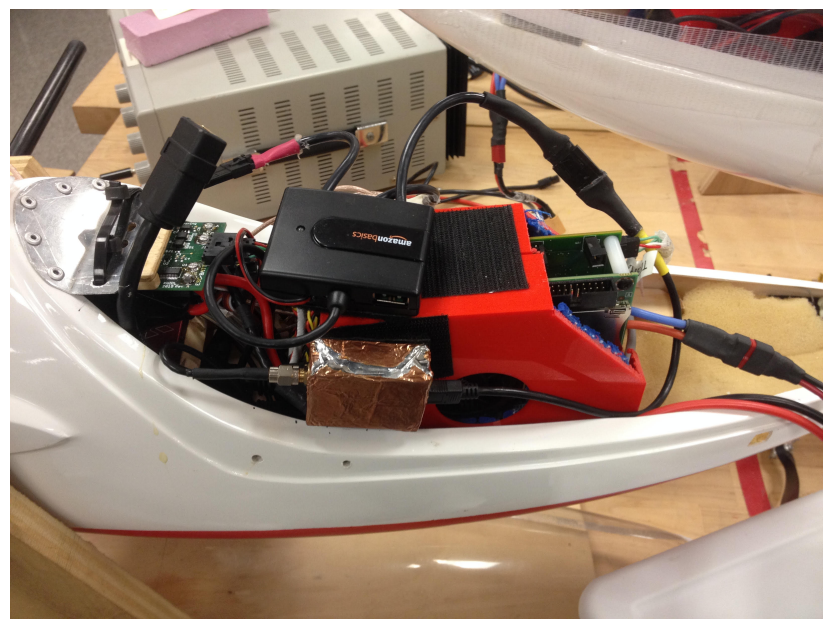

Figure B.4: Picture of our avionics containing a usb hub

In order to support all the usb connections, we use an non powered usb hub as shown in Figure B.4. The Vector Nav VN-200 sensor [8](GPS and IMU) draws current from the usb hub to operate. Since we were using a non powered hub, the current drawn by the hub from the processing board was not sufficient, the VN-200 sensor values had a huge variation for successive reading. This huge variation caused our autonomous controller to diverge ( $\mathrm{NaN}$ values). this issue was resolved by using a powered usb hub. Though the powered usb hub resolved the VN-200 sensor data issue, it produced another issue during flight tests. The aircraft that we used had small area to accommodate the avionics. Hence the avionics and the usb hub were placed very close to each other. The avionics and the usb hub inside the aircraft are covered using the aircraft's canopy. Somehow, this canopy put more pressure on the power cables to the usb hub and the power line to hub was broken. Since the hub was not powered the controller program did not receive any values from the sensors, that 
resulted in landing the aircraft. This issue was fixed by soldering the power cables.

We have encountered other hardware related issues, like battery used in the aircraft did not provide enough power to motor. This added difficulty in aircraft take off. Another issue that we faced is, the motor mount got broken while the aircraft was flying. The pitot tube, required for measuring the air speed, was not reading proper values. This happened because the tube was covered with frozen ice during the winter. 


\section{Appendix C}

\section{Avionics Installation}

We have included the instructions to install our avionics in this appendix. In this thesis, we used Tegra K1. We have included the bash commands detailed instructions on setting up the Tegra K1 processor.

\section{C.1 Software Dependencies}

1. OS - Ubuntu

2. Middleware - ROS

\section{C.2 Installing our Avionics Code}

All our code are maintained in our github repository [37]. Clone our repository inside the ROS workspace. After building the code, the avionics is ready to install into the aircraft. The description about each folder in our repository is as follows:

1. aircraft: This folder contains all the Matlab and generated $\mathrm{C}$ codes of our test aircrafts.

2. autopilot_releases: This folder contains all the Matlab files that are required for the simulation tests. 
3. launch: This folder contains all the ROS launch files that are created for each aircarft. This launch file calls all the required programs like sensor data reading, controller and ground station communication.

4. mavlink: We use mavlink protocol to communicate with the ground station. his folder contains library files for supporting mavlink.

5. msg: ROS using topics to publish and subscribe from other nodes. This folder contains the structure for all custom messages.

6. src: This folder contains all the source files. This includes the main controller program, reading sensor data, writing to servo and ground station communication. 


\section{Appendix D}

\section{Source Code Listing}

This appendix lists the source code for important programs we developed for the avionics.

\section{D.1 Controller Function Code}

This is the most important code. It subscribes all the sensor data and assigns it to Matlab generated variables. It calls the controller function and then it publishes the controller output.

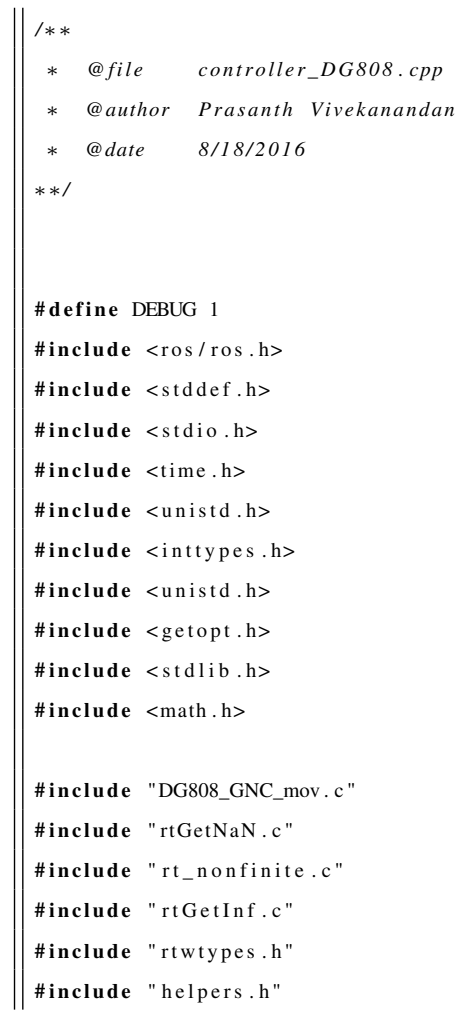


\#include < drone_ros/ins.h>

\#include <drone_ros/pwm.h $>$

\#include $<$ drone_ros/data.h $>$

\#include < drone_ros/way.h $>$

\#include < drone_ros/hil_data.h>

//messgae library for GPS position

\#include "sensor_msgs/NavSatFix.h"

//message library for GPS velocity

\#include "geometry_msgs/Twist.h"

//message library for $P Q R$ and acceleration

\#include "sensor_msgs/Imu.h"

//message library for Phi, Theta and Psi

\#include "geometry_msgs/Point.h"

\#include "sensor_msgs/Temperature.h"

\#include "sensor_msgs/FluidPressure.h"

\#include "sensor_msgs/BatteryState.h"

$/ * * * * * * * * * * * * * * * * * * * * * * * * * * * * * * * * * * * * * * * * * * * * * * * * * * * * * * * * * * * * * * * * * * * * * * * * * *$

* Public Definitions

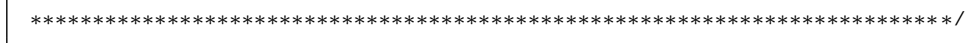

\#define PDEBUG(fmt, ...)

do $\{$ if (DEBUG) fprintf(stderr, fmt, _VA_ARGS_ $) ;\}$ while (0)

$/ * * * * * * * * * * * * * * * * * * * * * * * * * * * * * * * * * * * * * * * * * * * * * * * * * * * * * * * * * * * * * * * * * * * * * * * * * * * * *$

* Public Types

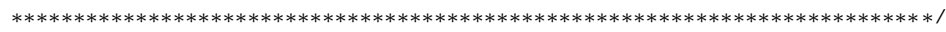

\#define SAVEFILE 1

\#define DEG2RAD 0.0174532925

\#define GRAVITY 9.8

\#define DENSITY 0.002295

$/ * * * * * * * * * * * * * * * * * * * * * * * * * * * * * * * * * * * * * * * * * * * * * * * * * * * * * * * * * * * * * * * * * * * * * * * * * * *$

* Global Variables

***********************************************************************************I

ros:: Publisher g_pub_data_arduino;

ros: : Publisher g_pub_data_gs;

ros:: Publisher g_pub_data_hil; //used to publish data to the Hardware In the Loop node

void call_controller();

std:: string g_controller_frame_id;

struct usb_status_struct usb_status; // Stores the health condition of USB devices ; Used by the decision logic block in the auto generated code

struct pwm_conversion_struct pwm_conversion_factors; //Structure to store the conversion factors for each surfaces

struct helpers_struct helpers; // To be used in ROS subscription in log node and controller node

ExtU_DG808_GNC_mov_T $*$ controller_in $=$ \&DG808_GNC_mov_U;

ExtY_DG808_GNC_mov_T $*$ controller_out $=$ \&DG808_GNC_mov_Y;

$/ * * * * * * * * * * * * * * * * * * * * * * * * * * * * * * * * * * * * * * * * * * * * * * * * * * * * * * * * * * * * * * * * * * * * * * * * * *$

* Public Function Prototypes

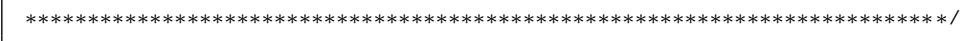

void send_pwm(); 


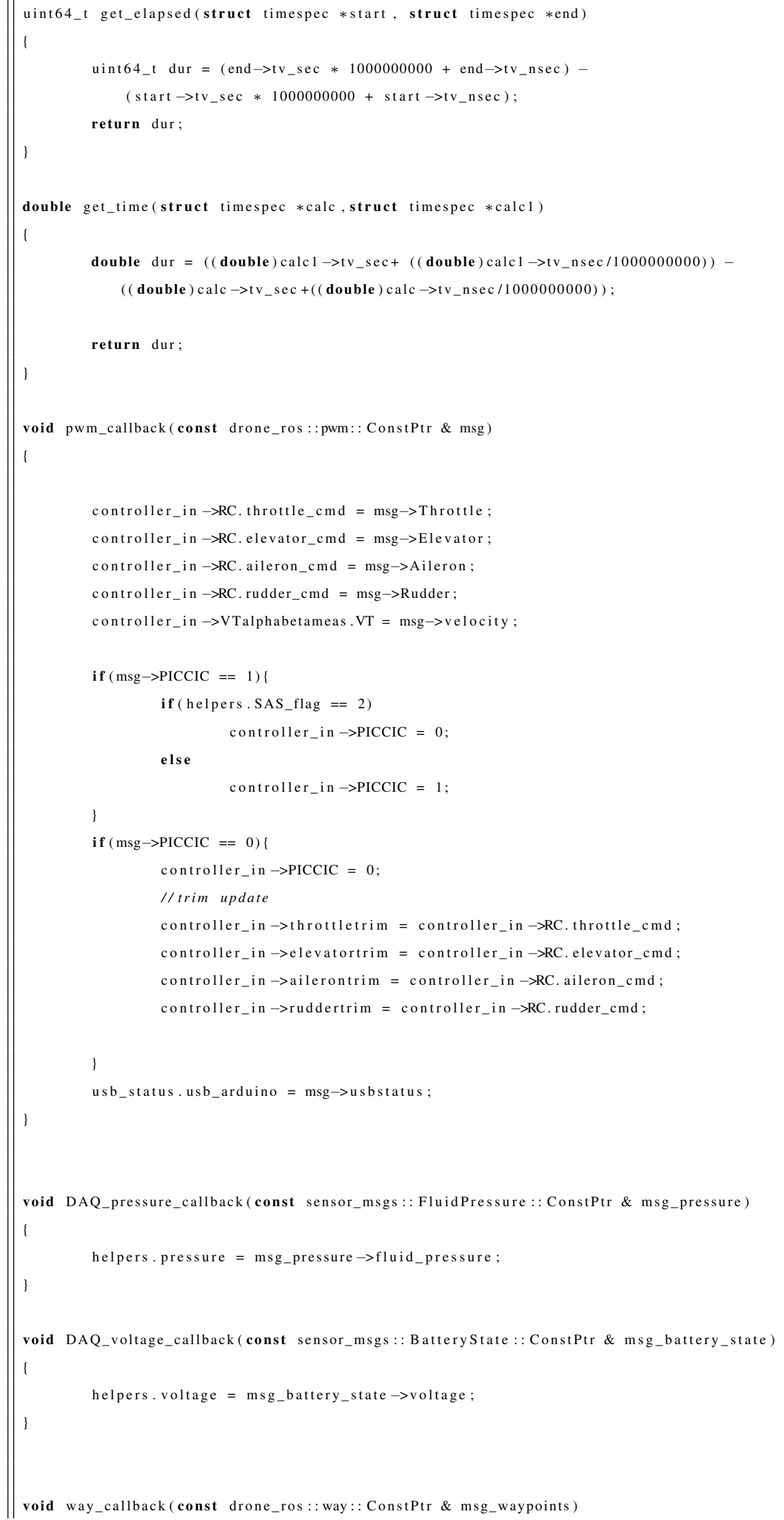




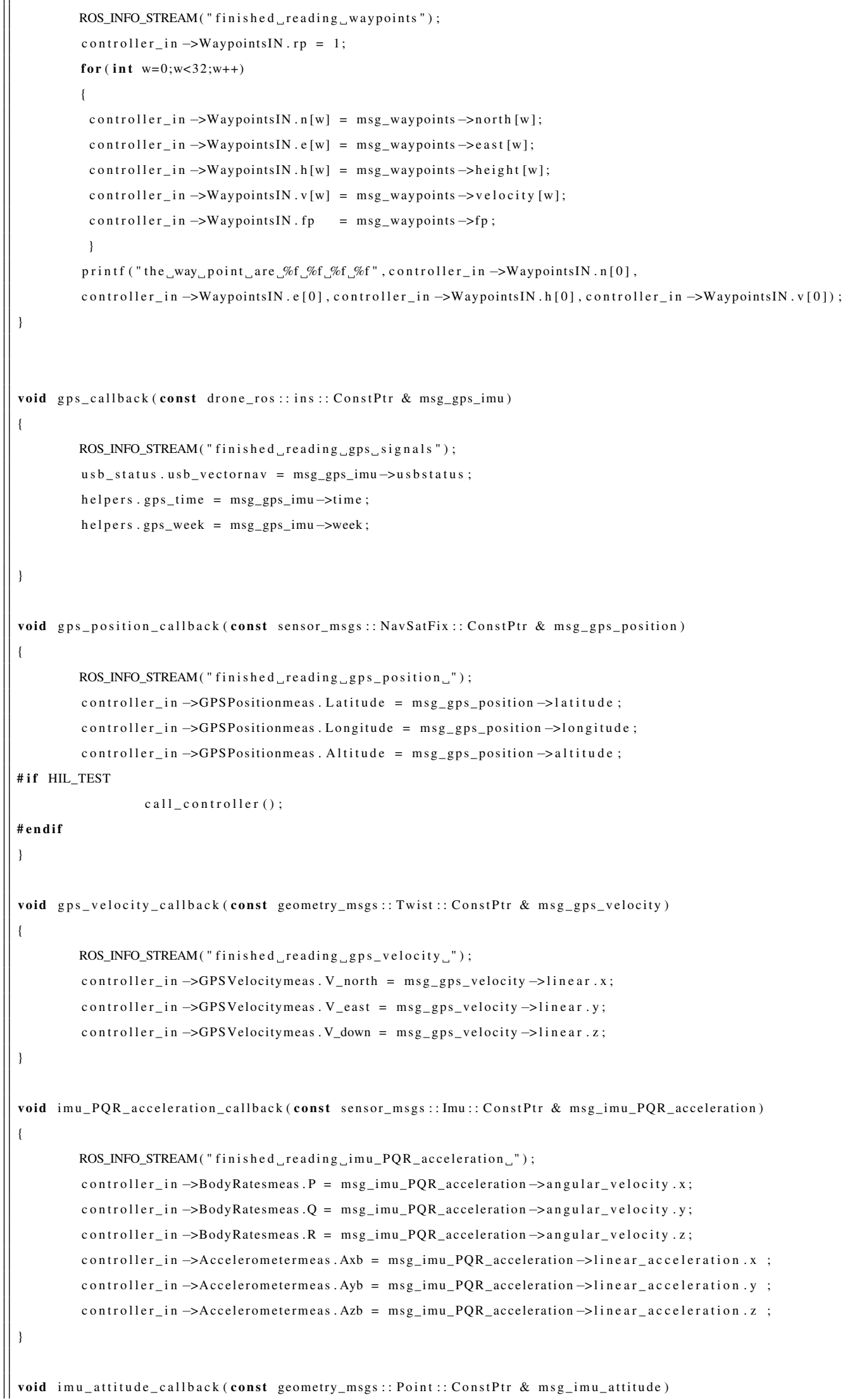




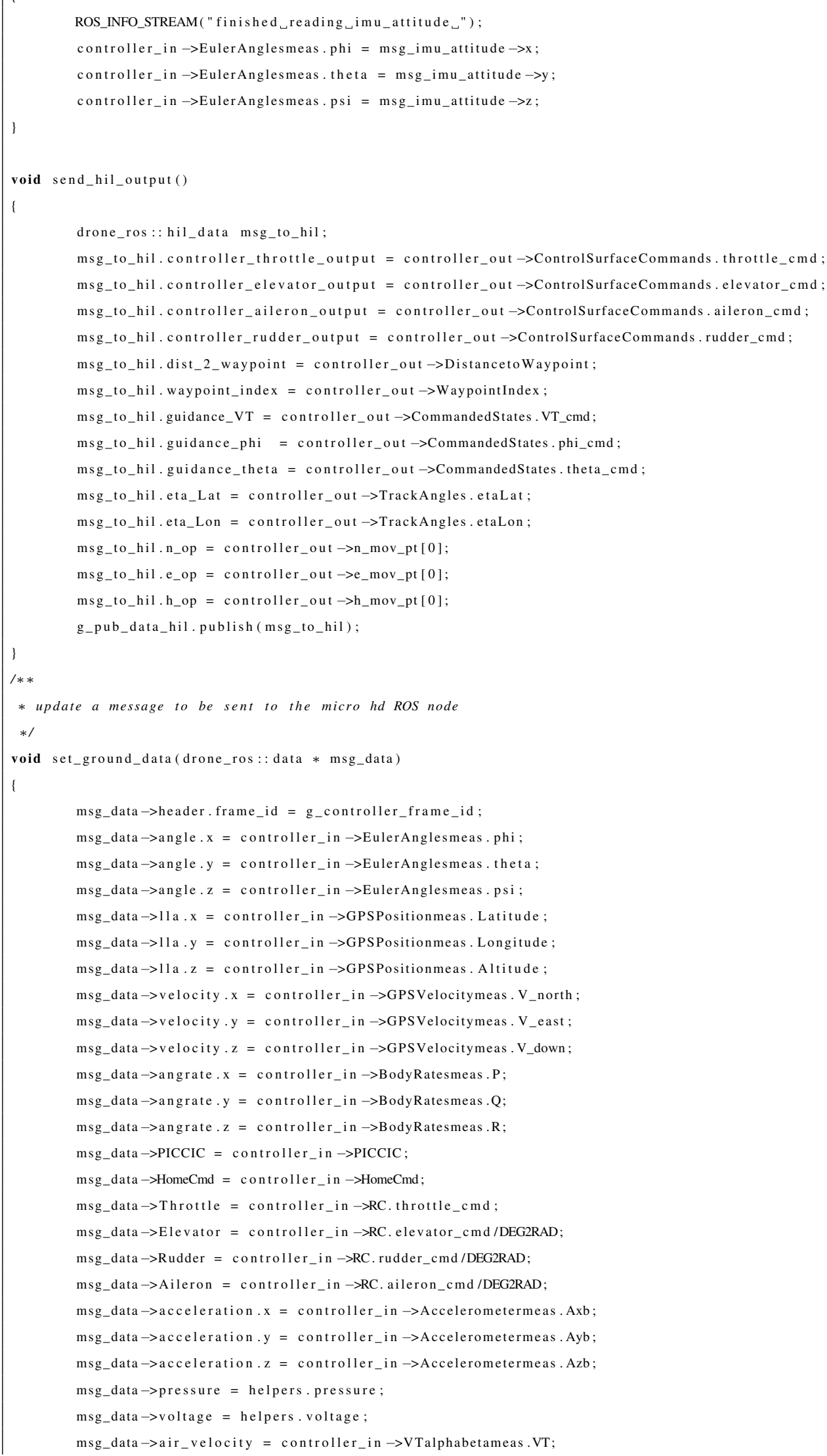




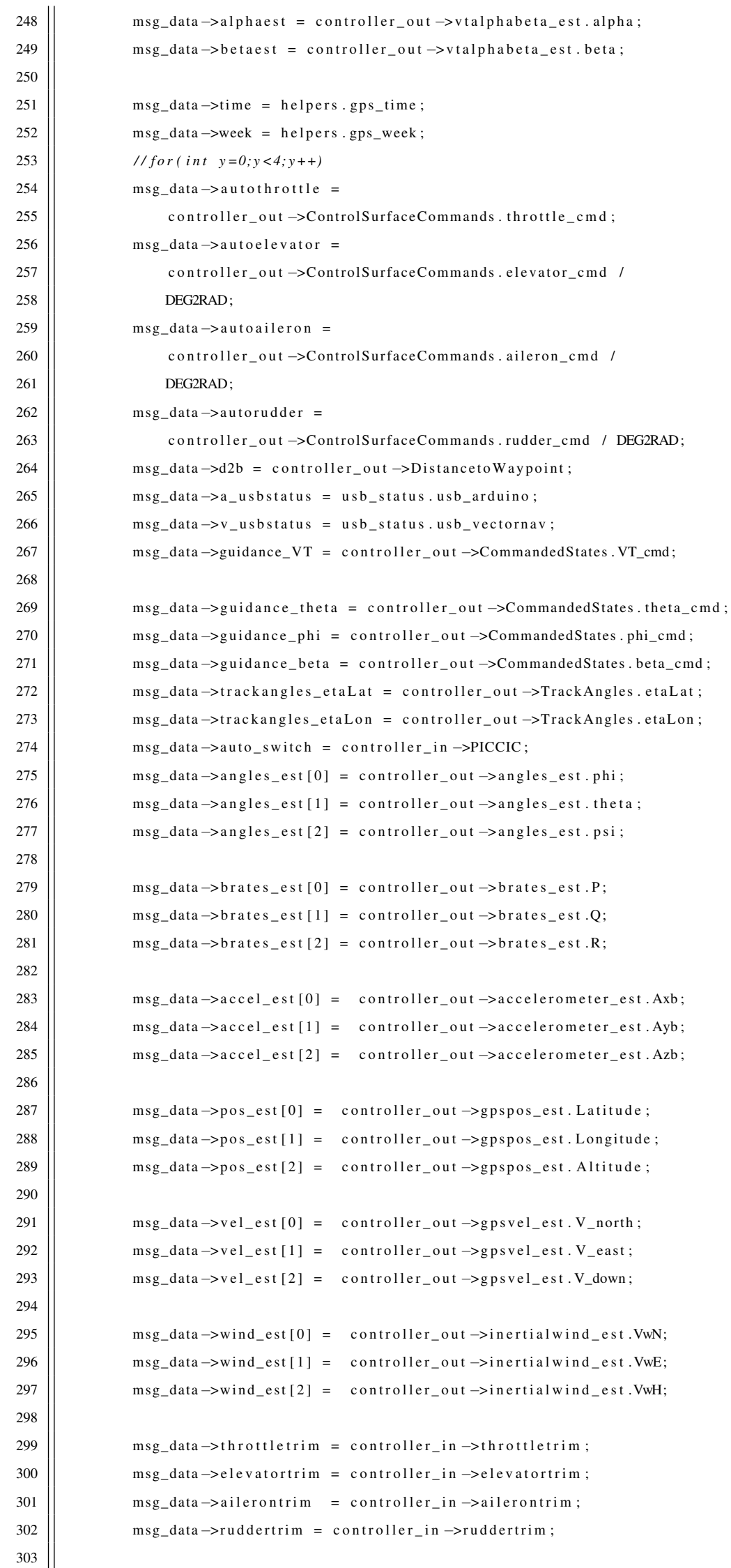




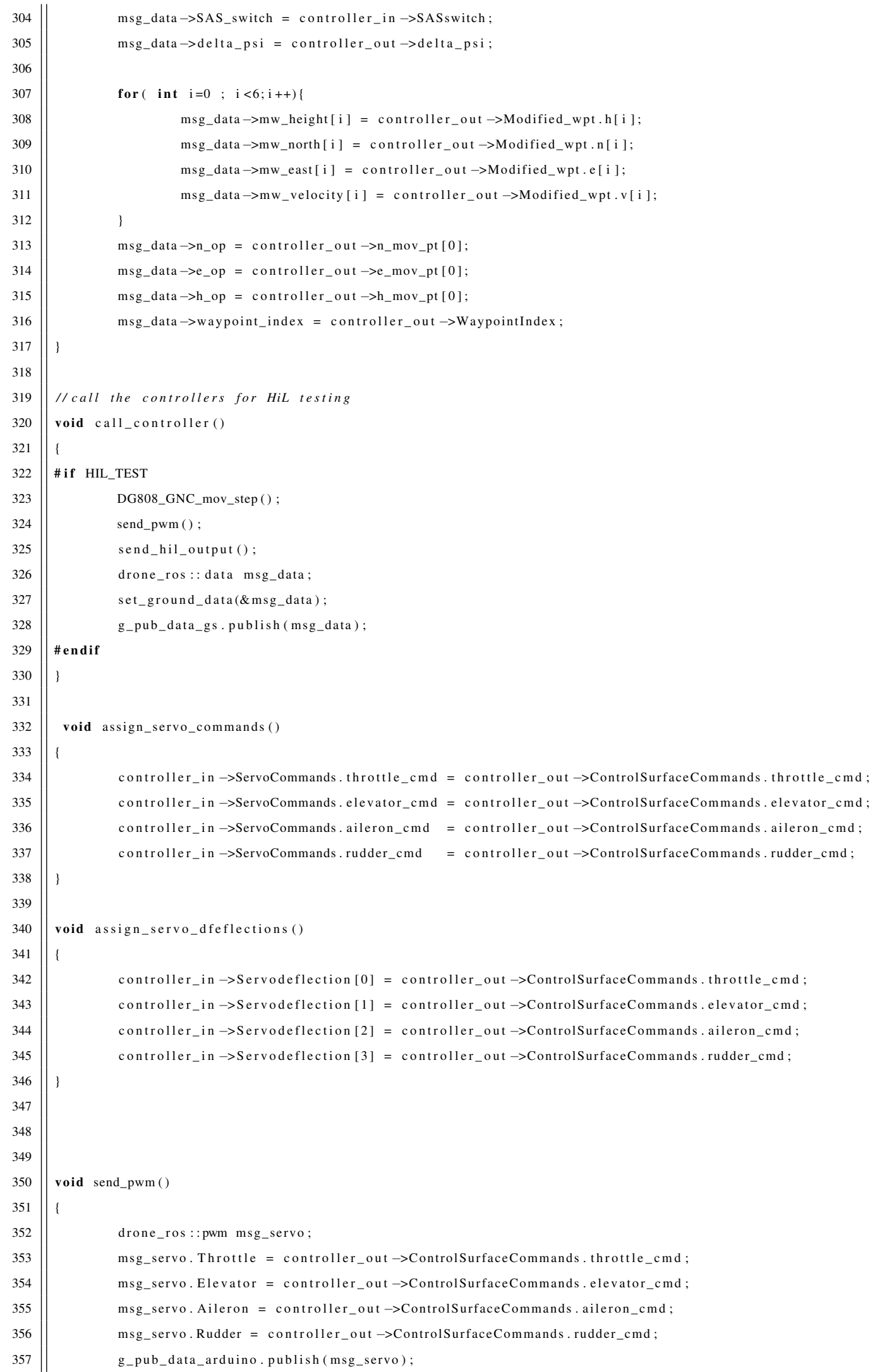




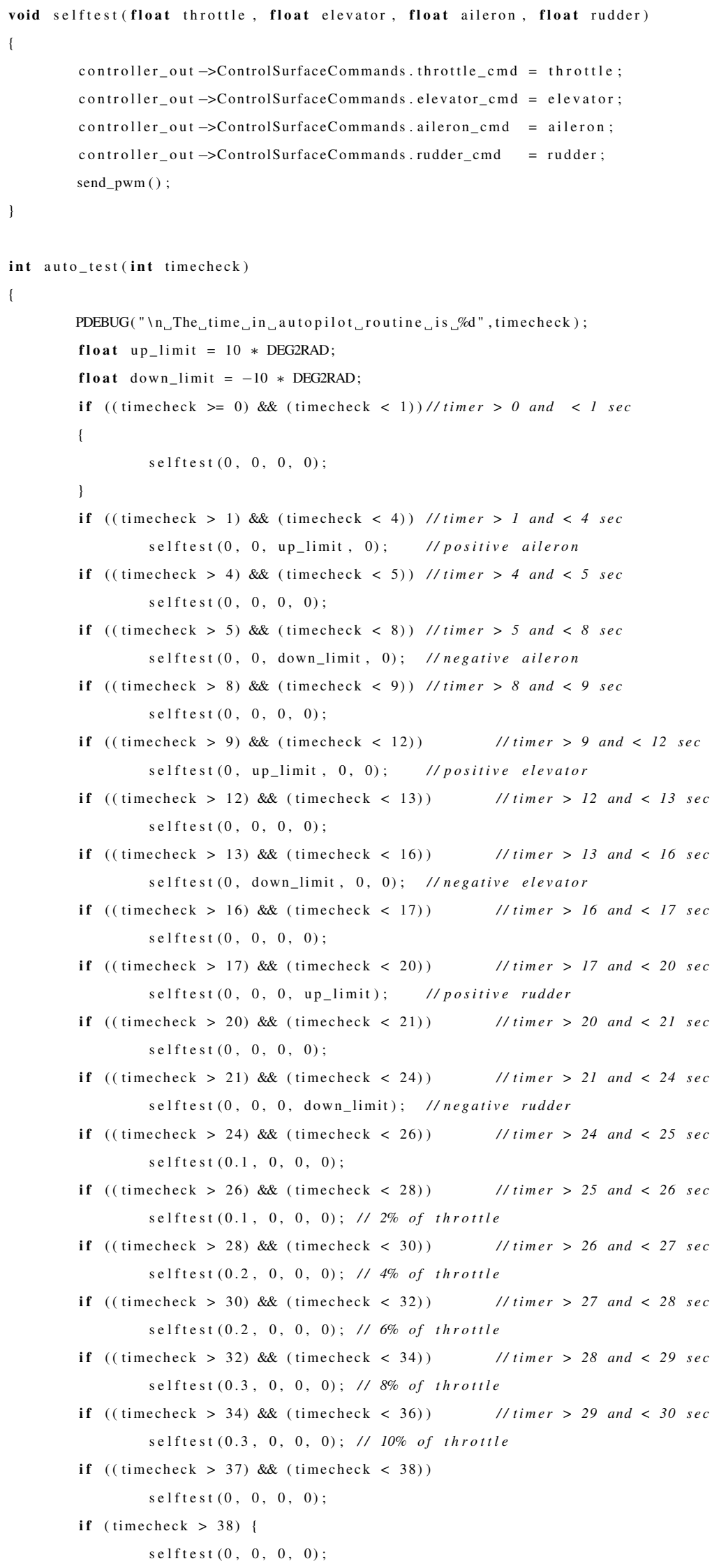




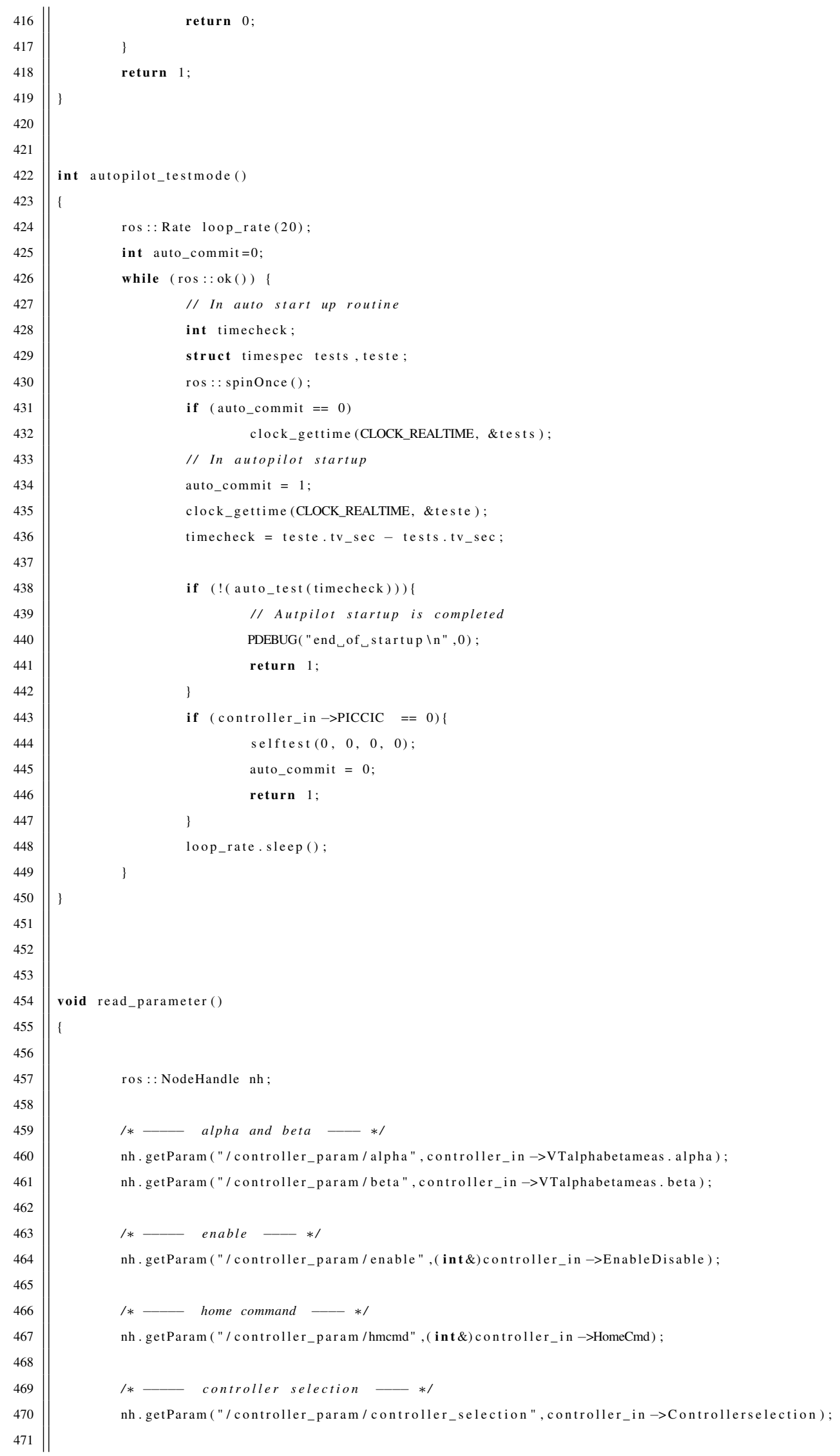




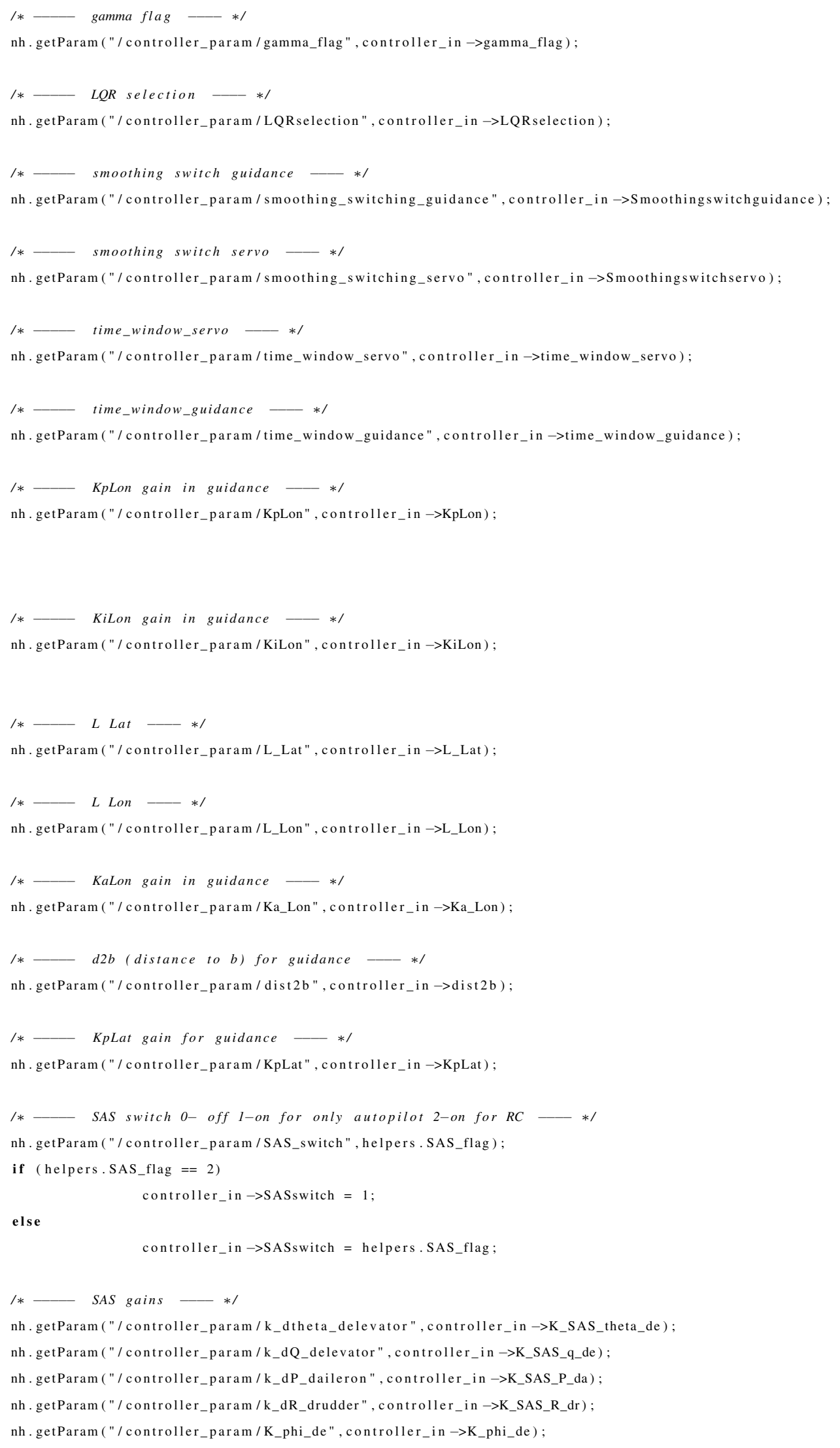




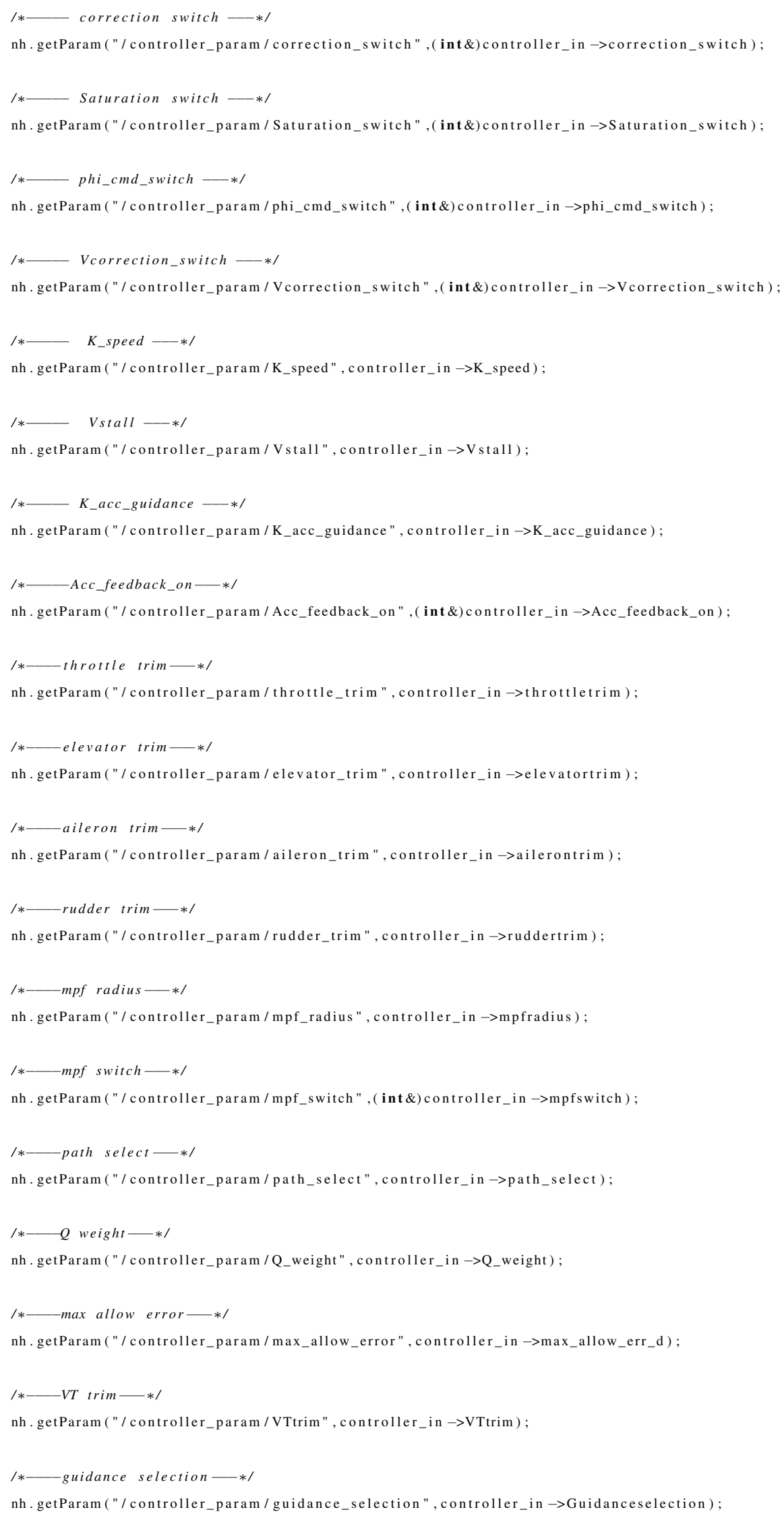




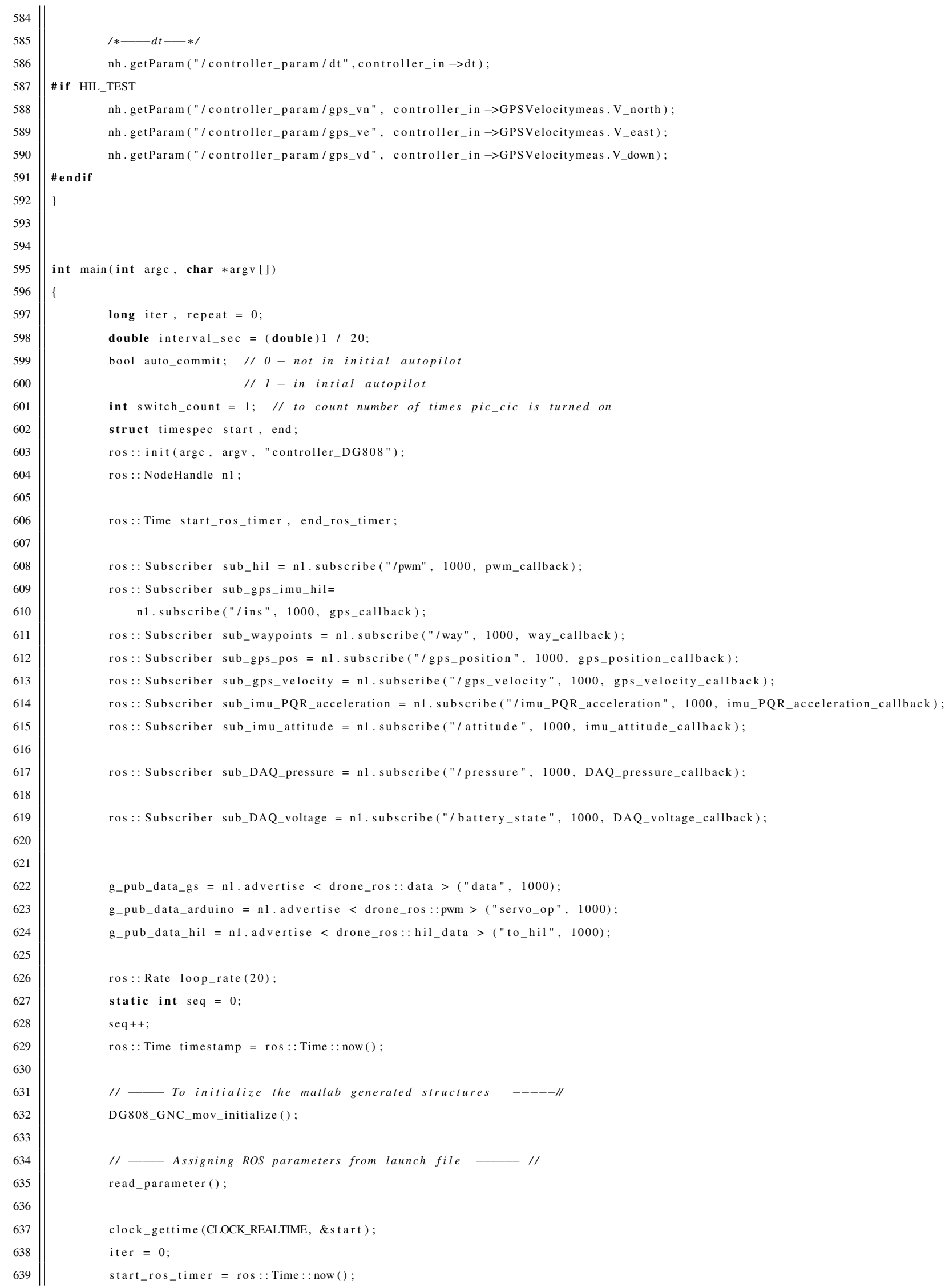




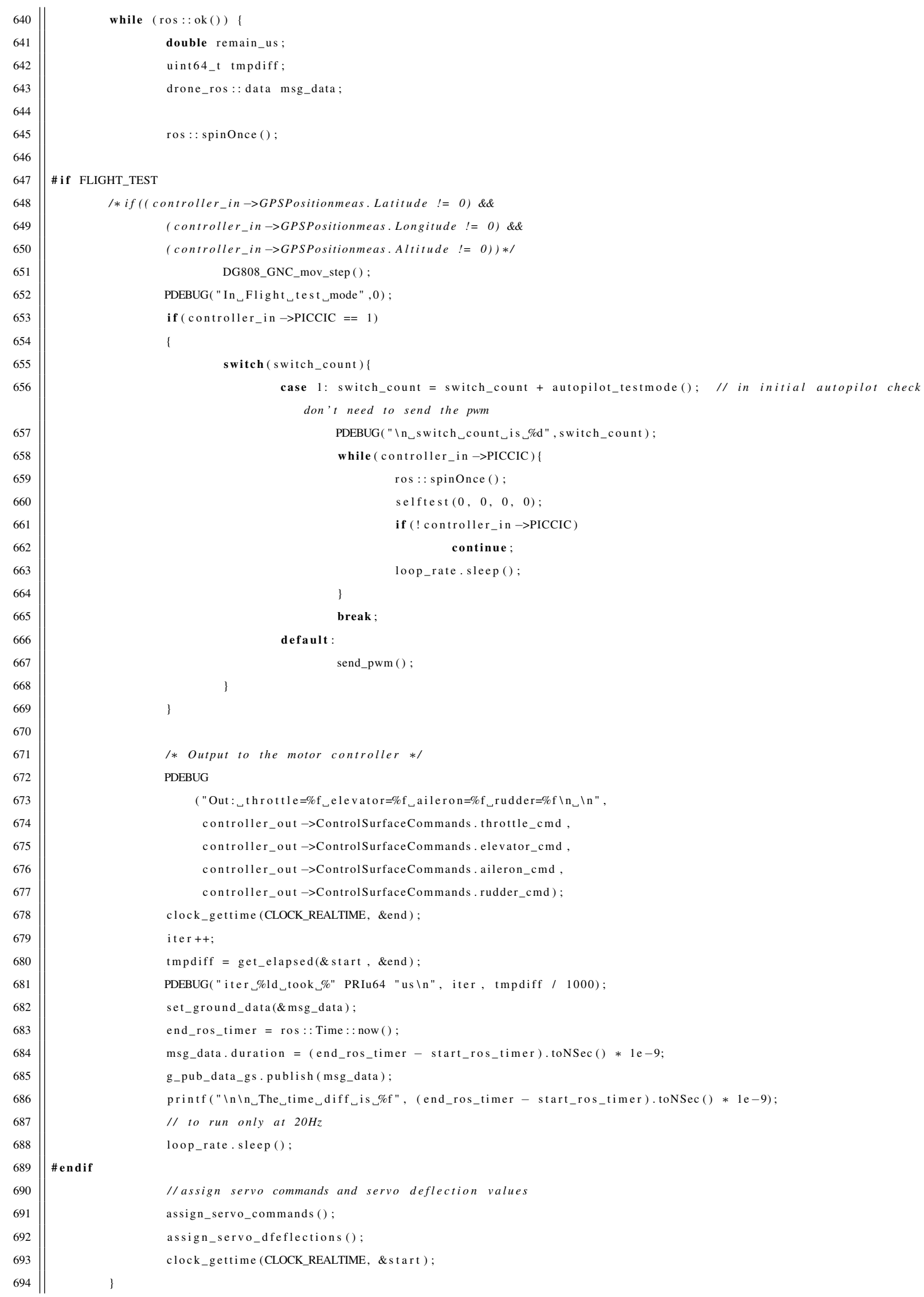


$\left.\begin{array}{l|l}695 \\ 696 \\ 697\end{array} \|\right\} \quad \begin{aligned} & \text { DG808_GNC_mov_terminate () } \\ & \text { return } 0\end{aligned}$

\section{D.2 Sensor Data Acquisition Code}

This section lists the code to read all sensor data and output the servo commands to the motors.

\section{D.2.1 RC, Air Velocity and Battery Voltage}

This sub section contains the code to read RC, air velocity and battery voltage from our custom built avionics board. This also contains code to send values to the actuator.

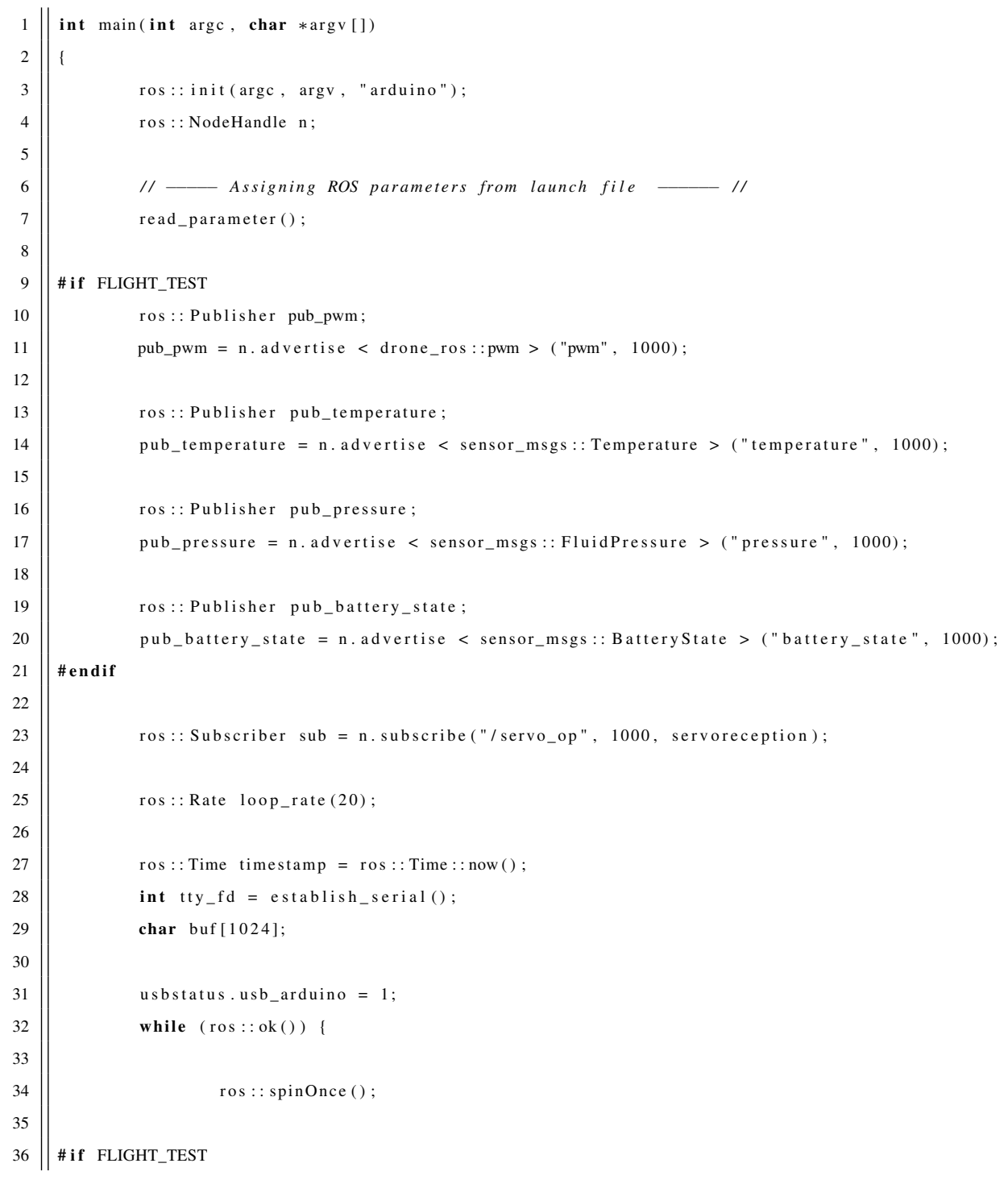




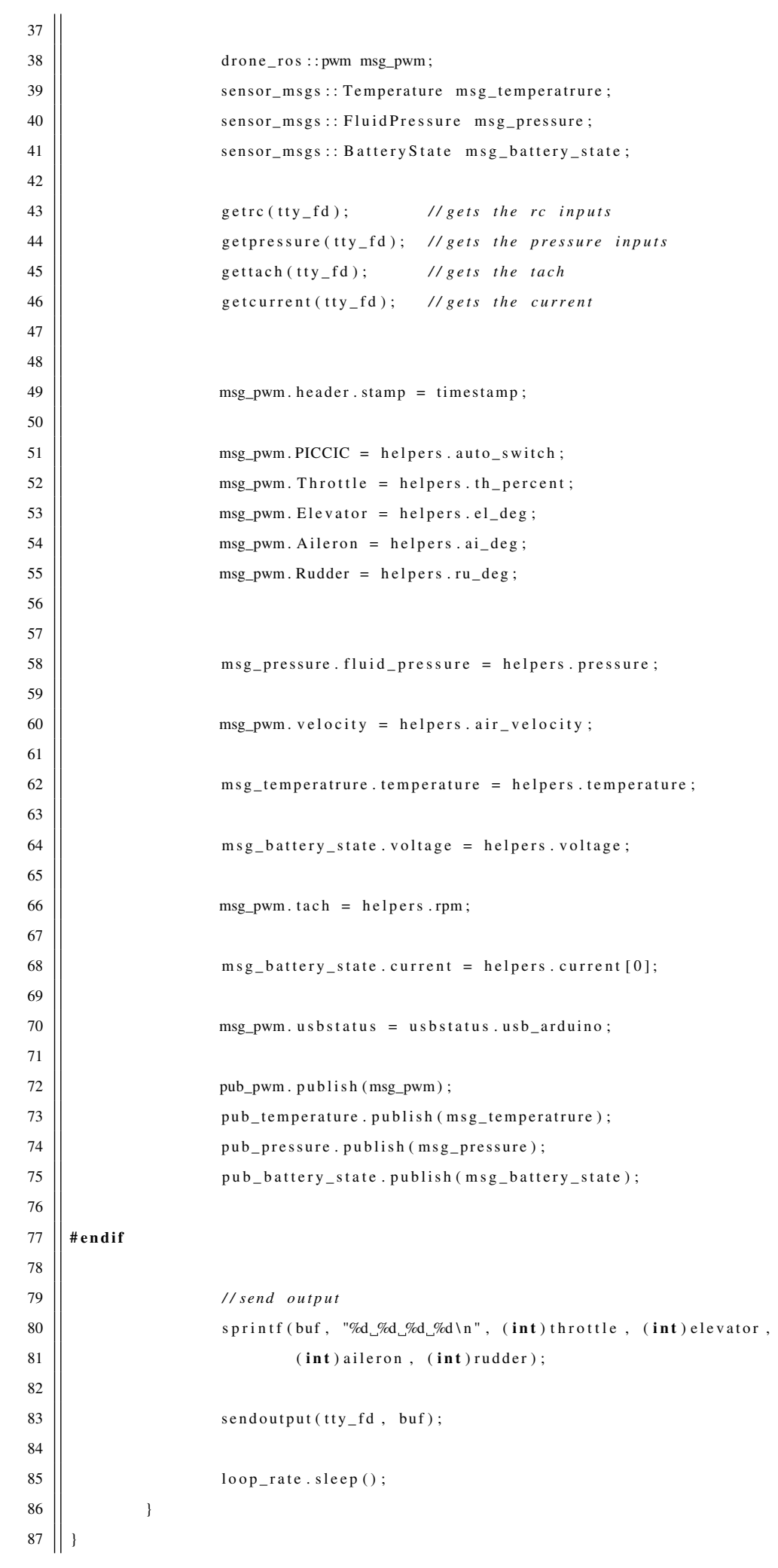




\section{D.2.2 GPS and IMU}

We have used Vector Nav VN 200 [8] for measuring position and attitude. The following code is to read from VN200 sensor.

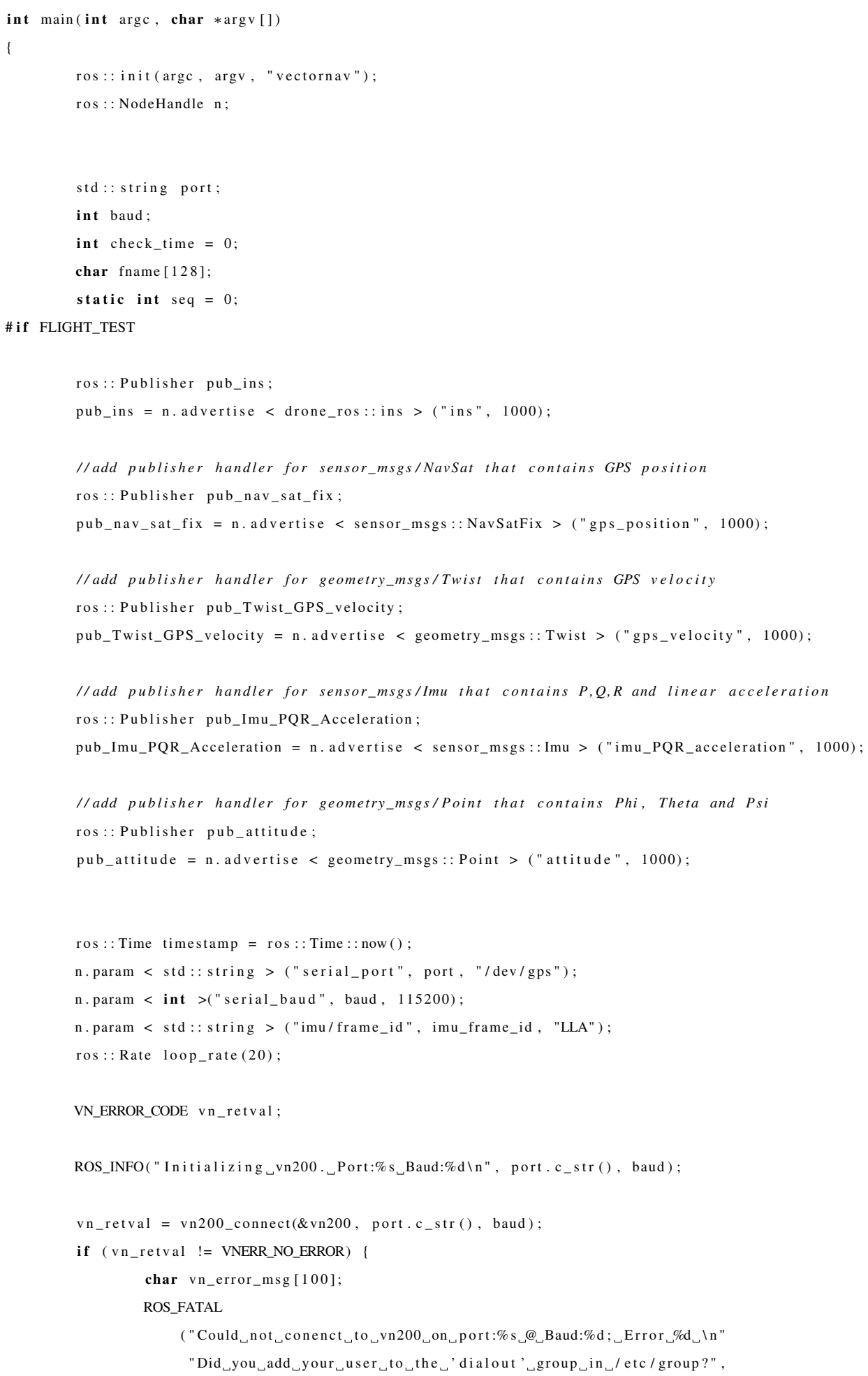




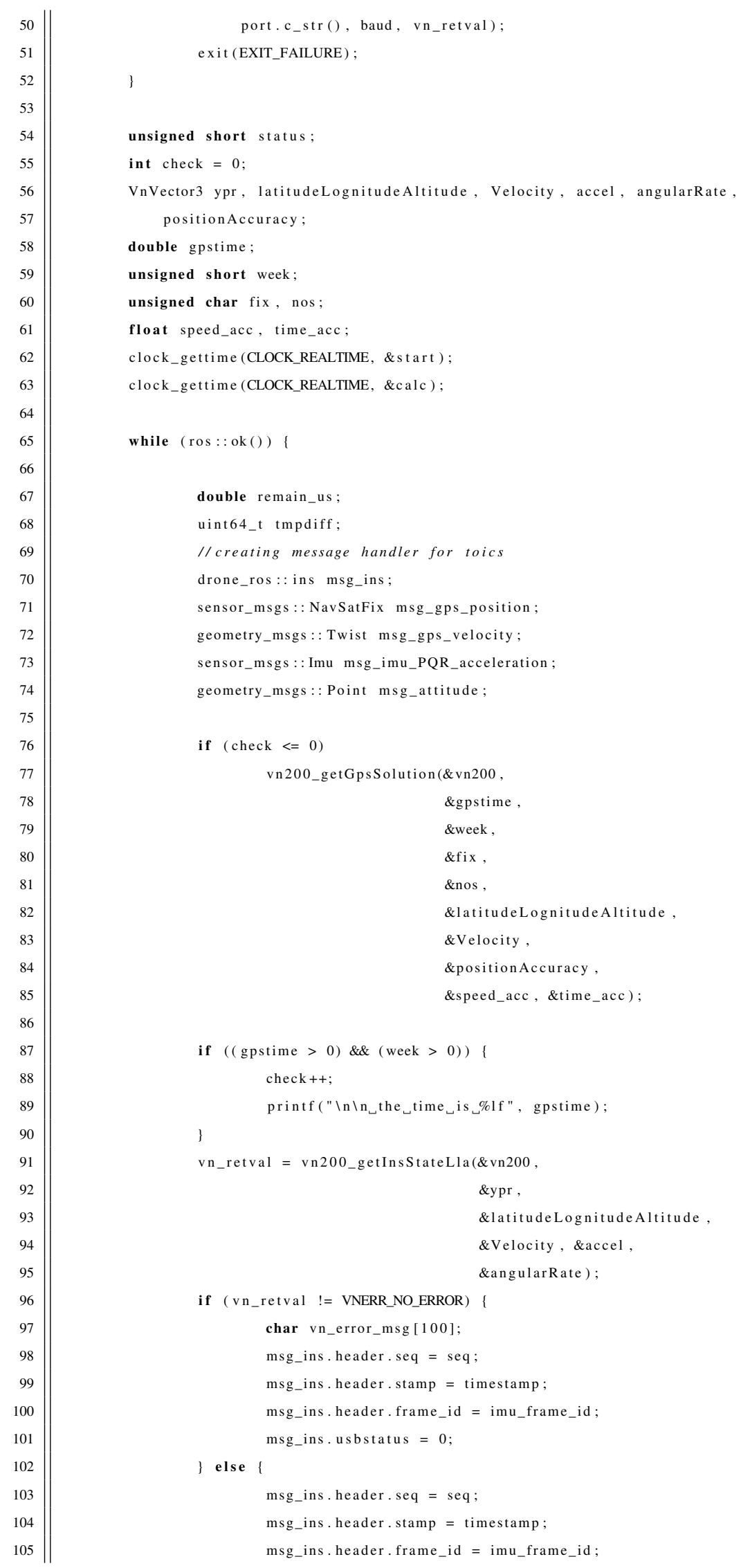




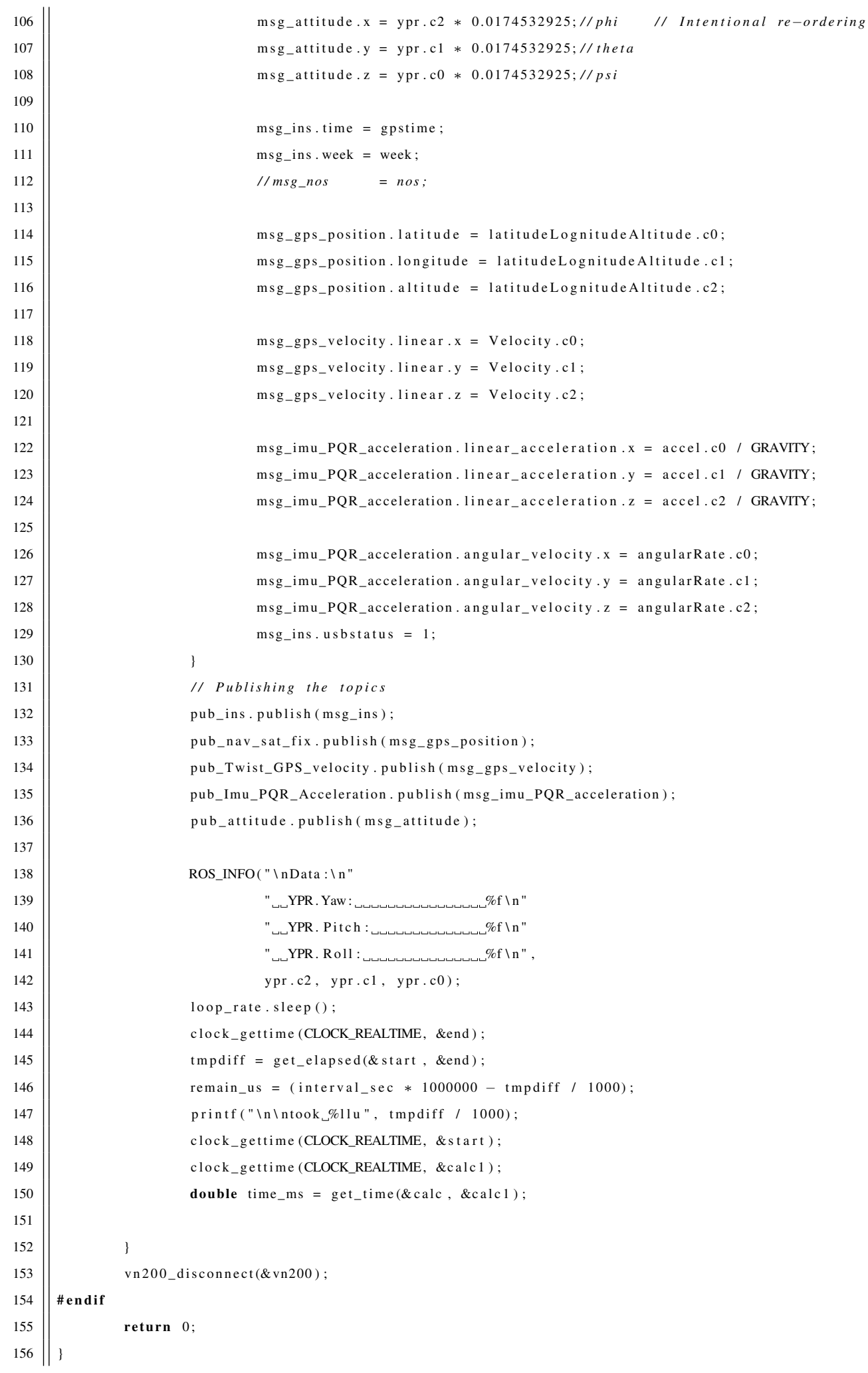

